\title{
Electric-field induced transitions in suspensions of charged colloidal rods
}

\author{
Kyongok Kang* and Jan K. G. Dhont \\ Received 21st May 2009, Accepted 15th October 2009 \\ First published as an Advance Article on the web 27th November 2009 \\ DOI: $10.1039 / b 910046 f$
}

We explore transitions in suspensions of fd virus at a low ionic strength, induced by external electric fields at frequencies where double layers are polarized. On the basis of the different optical morphologies, phase/state diagrams are constructed in the field-amplitude versus frequency plane and the field-amplitude versus concentration plane. Due to interactions between polarized double layers, for low frequencies, various phases and dynamical states are found: a nematic phase, a striped phase and a dynamical state where nematic domains melt and reform. At relatively high frequencies of a few $\mathrm{kHz}$, a uniform homeotropic phase is induced. The various phases and states are characterized by means of polarization microscopy, birefringence, dynamic light scattering and video-correlation spectroscopy. An expression is derived for the attenuation of the electric field due to electrode polarization, which is tested experimentally. This theory is used to correct phase/state diagrams for electrode polarization.

\section{Introduction}

The response of many soft matter systems to an external electric field results from permanent or dielectrically induced macromolecular dipoles. The latter type of polarization is responsible for field-induced structural transitions in concentrated suspensions of colloidal spheres ${ }^{1-3}$ and low-aspect ratio rods. ${ }^{4}$ Here, the colloidal cores are dielectrically polarized, resulting in interacting electrical dipoles that lead to anisotropic structures like strings and sheets of colloidal particles. The frequencies that are used for such suspensions are in the MHz-range. At these high frequencies the electric double layers of the colloidal particles are not polarized. Until now, there are only a few experimental studies that address effects of double-layer polarization of interacting colloids. Double-layer polarization only occurs at sufficiently low frequencies, below a few $\mathrm{kHz}$. As far as we know, the first experiments in electric fields where double-layer polarization is responsible for the observed response have been performed on suspensions of fd-virus particles by the Konstanz group. ${ }^{5}$ For concentrations larger than a few times the overlap concentration, the alignment is found to change from parallel to the electric field to an anomalous perpendicular orientation, depending on the field amplitude and frequency. This anomalous orientation is entirely the result of interactions between the polarized double layers. The applied field amplitudes are too small to induce sufficiently large dipoles in the dielectric core of the fd-virus particles that could lead to additional dipole-dipole interactions. A similar behaviour has been found in Ref. [6] for mixtures of rod-like and spherical colloids. The anomalous orientation in these systems is attributed to a possible asymmetric crowding of spheres, giving rise to electro-osmotic flows that induce a torque on the rods leading to perpendicular alignment. An anomalous orientation of a single flexible, charged rod in an electric field is

Forschungszentrum Jülich, Institute für Festkörper Forschung (IFF), Weiche Materie, D-52425 Jülich, Germany. E-mail: k.kang@fz-juelich. de; Web: http://www.fz-juelich.deliffld_iwm found in simulations. ${ }^{7}$ The perpendicular orientation is due to the fact that for a bent rod, the center-of-force due to hydrodynamic friction no longer coincides with that of the electric forces. This leads to a torque that orients the rod perpendicular to the electric field. In the experiments in Ref. [5], however, the observed anomalous orientation has a different origin, and is clearly due to interactions between the rods. So far it is not understood why interactions between polarized double layers of rods give rise to an orientation perpendicular to the electric field.

In this paper we study the response of concentrated suspensions of colloidal rods (fd-virus particles) to external electric fields at frequencies where electric double layers are polarized. The concentrations are about thirty times larger than the overlap concentration, and are such that in the absence of an electric field there is nematic-isotropic coexistence. At these high concentrations, and for the fixed low ionic strength, we observe several phase/state transitions depending on the field amplitude and frequency. The transitions are reported briefly in Ref. [8] without detailed characterization. These transitions have not been observed for the suspensions with much lower concentrations investigated by the Konstanz group, ${ }^{5}$ nor in the electric-field relaxation experiments in Ref. [9]. It should be mentioned that anchoring conditions at the electrode surfaces play no role in the Konstanz experiments nor in our experiments. We make observations in the bulk part of a wide sample cell. As far as we know, fd-virus suspensions have been used for the first time as model colloids in Ref. [10], where the response to magnetic fields is investigated. The magnetic field acts diamagnetically with a torque on each individual fd-virus, which is an entirely different mechanism as compared to the electric field.

A theory that explains the response of concentrated suspensions to alternating electric fields at low frequencies does not yet exist. Such a theory should describe (i) the double-layer structure in alternating electric fields, (ii) colloid-colloid interactions due to polarization of double layers, (iii) colloid-colloid interactions due to electro-osmotic flows which are generated within the deformed 
double layers, and (iv) the collective phenomena that arise due to the electric-field induced colloid-colloid interactions. Doublelayer polarization has been extensively studied for a long time in connection to electrophoresis. These theories are limited to spherical colloids (see, for example, the seminal papers by Overbeek, ${ }^{11}$ Booth, ${ }^{12}$ DeLacey ${ }^{13}$ and O'Brien ${ }^{14}$ ). Polarization of rod-like colloids by steady electric fields has been discussed in Refs. [15],[16]. Polarization of non-spherical particles by alternating fields has, as far as we know, only been analyzed, in part numerically and for thin double layers, in Ref. [17]. So far, there is no analytical result available for the charge distribution around a rod-like macro-ion for arbitrary Debye screening lengths in alternating electric fields that would allow the construction of a theory that describes the response of concentrated suspensions to external electric fields. Polarization contributions of condensed ions on a rod, which are analyzed in Refs. [18],[19], may also play a role. It is still an open question as to what the relative contributions to the total polarization of the diffuse double layer and the layer of condensed ions are.

\section{Fd-virus suspensions and the electrical sample cell}

The suspensions of fd-virus were prepared and purified following standard biological protocols, using the XL1 blue strain of E. coli as the host bacteria. ${ }^{20} \mathrm{Fd}$-viruses are stiff, rod-like particles with a length of $880 \mathrm{~nm}$ and a core diameter of $6.7 \mathrm{~nm}$. The persistence length is about $2200 \mathrm{~nm}$. The surface charge of fd-virus particles as obtained from titration curves is approximately $-8700 e$ at $p H=6.9 .^{21}$ The fd-suspensions are prepared by dialyzing for 2 days against a TRIS/HCl-buffer with an analytical concentration of $1.6 \times 10^{-4} \mathrm{M}$. The appropriate concentrations are then obtained by dilution with the same buffer. For such a low buffer concentration, the amount of carbon dioxide that dissolves from the air must be taken into account for the calculation of the ionic strength and $p H$ (this is discussed in appendix D of Ref. [22]). Carbon dioxide increases the ionic strength from $0.7 \times 10^{-4} \mathrm{M}$ to $1.5 \times 10^{-4} \mathrm{M}$, and decreases the $p H$ from 8.2 to 6.9 . The corresponding Debye length is $27 \mathrm{~nm}$, which is large in comparison to the rod diameter. The concentration of fd virus is varied from $1.2 \mathrm{mg} / \mathrm{ml}$ to $4.0 \mathrm{mg} / \mathrm{ml}$.

A home-made optically transparent electrical cell is used to facilitate imaging through a microscope, birefringence measurements and dynamic light scattering experiments. We use commercially available custom-designed indium-tin-oxide (ITO) coated float glass (from Präzisions Glas und Optik $\mathrm{GmbH}$, CEC500S) of dimensions $40 \times 70 \mathrm{~mm}^{2}$ with a thickness of $0.7 \mathrm{~mm}$. The ITO layer has a high visible light transmission $(90 \%)$ at $633 \mathrm{~nm}$, and the coating thickness is $15 \mathrm{~nm}$. A sketch of the cell in combination with the microscope is given in Fig. 1. $400 \mu \mathrm{l}$ of fd-suspension is loaded on the bottom plate within a rectangle of insulating PTFE film-spacer (Armbrecht and Matthes $\mathrm{GmbH}$, AR5038 and AR5038GP). The upper electrode is then gently placed onto the lower plate. Due to capillary forces, the sample droplet adopts a circular form, the radius of which is typically 9-10 $\mathrm{mm}$. The precise sample thickness is calculated from this radius, which is in the range of $1.40 \pm 0.15 \mathrm{~mm}$. The two plates are then sealed with the same PTFE tape in order to avoid evaporation and to fixate the two electrodes. The ITO coatings are located on the side of the sample. All measurements are

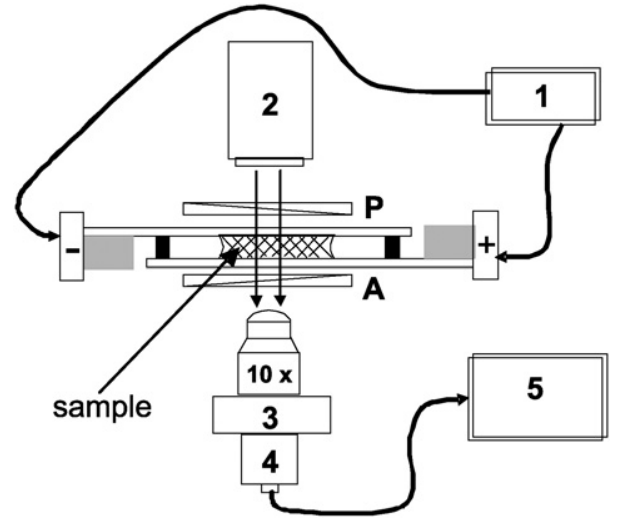

Fig. 1 A schematic drawing of the electrical cell in combination with the microscope: (1) function generator, (2) white-light source, (3) PlasDIC filter (Carl Zeiss), (4) CCD camera and (5) PC interface. P and A are crossed polarization sheets. The insulating spacer is depicted as the black solid regions at both electrodes, and the arrow indicates the location of the sample that is held in place by surface-tension forces.

performed at the center of the sample to ensure a homogeneous electric field. The ITO layers are then connected to a function generator (Avtech model AV-151G-B, 1-350 kHz, maximum $\pm 200 \mathrm{~V}$, load resistance $\geq 50 \mathrm{k} \Omega$ ) by means of electronic connection pins. A sinusoidally varying electric potential is applied to the electrodes.

Images are recorded with an inverted microscope (Carl Zeiss, Axiovert 40CFL model) equipped with a CCD camera (AxioCam Color A12-312, $1300 \times 1030$ pixels). Images are taken in polarization mode in combination with Differential Interference Contrast. Since the dimensions of morphologies are of the order of tens of microns, and in order to view a relatively large field of view of typically $900 \times 700 \mu \mathrm{m}^{2}$, images are collected using a $10 X$ objective lens (NA 0.30 EC Plan-Neofluar).

\section{Electric phase/state diagrams}

In this section we discuss the morphologies that are observed by means of polarization microscopy. Various phases and dynamical states are identified, of which the characterization will be discussed in section IV.

Phase/state diagrams are constructed for the two fd-virus concentrations $2.0 \mathrm{mg} / \mathrm{ml}$ and $2.8 \mathrm{mg} / \mathrm{ml}$ in the applied electric field amplitude versus frequency plane. These concentrations are about 26 and 37 times larger than the overlap concentration, respectively. The phase/state diagrams for these two fd-virus concentrations in the field amplitude, $E_{0}$, versus frequency, $\nu$, plane are given in Fig. 2. The solid lines are phase-transition lines while the dashed lines are lines where qualitative changes either in structure or dynamics are observed. In order to define the various transitions, we shall first discuss what is observed when the applied field strength $E_{0}$ is increased at a fixed, low frequency of $10 \mathrm{~Hz}$ for [fd] $=2.8 \mathrm{mg} / \mathrm{ml}$ (see Fig. $2 b$ ).

Without an applied field, the equilibrium state is a nematic in coexistence with an isotropic phase, which we refer to as the $N$-phase. A typical depolarized microscopy image of this phase is given in Fig. 3. On increasing the field amplitude to about $1 \mathrm{~V} / \mathrm{mm}$, a striped texture is observed, in addition to the 

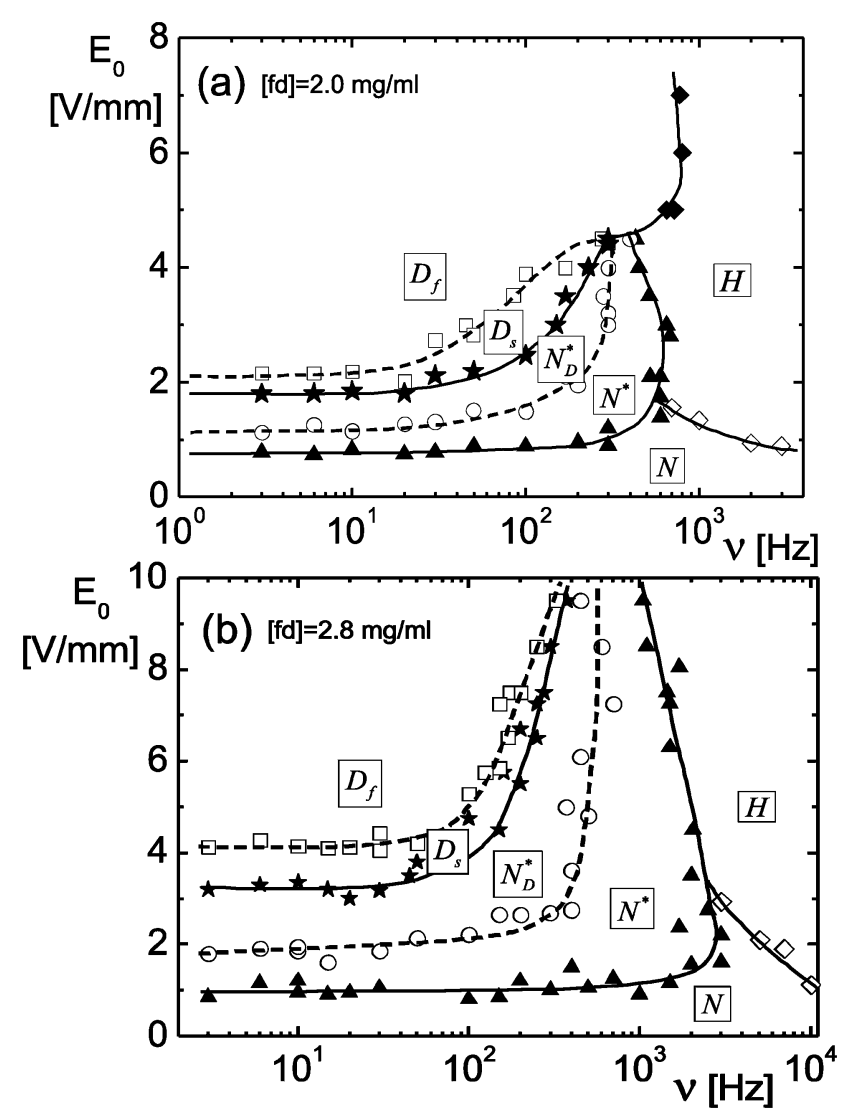

Fig. 2 The electric phase/state diagram of fd-virus suspensions in the field-amplitude versus frequency plane for fd concentrations of (a) 2.0 $\mathrm{mg} / \mathrm{ml}$ and (b) $2.8 \mathrm{mg} / \mathrm{ml}$. The solid lines refer to phase/state transitions, while the dashed lines refer to more gradual transitions involving the texture and dynamics of $N$-domains. The symbol $\boldsymbol{\Delta}$ refers to the $N$-to $N^{\star}$ transition, $\bigcirc$ to the $N^{\star}$-to- $N^{\star}{ }_{D}, \star$ to the $N^{\star}{ }$-to- $D_{s}, \square$ to the $D_{s}$-to- $D_{f}$, $\diamond$ to the $N$-to- $H$, and $\diamond$ to the $D_{f}$-to- $H$ transition.

$N$-domains, as can be seen in Fig. 3. As will be discussed in section IV A, there are strong indications that this striped texture corresponds to chiral-nematic domains, which is the reason that we refer to this phase as the $N^{*}$-phase. On further increasing the field amplitude above $2.0 \mathrm{~V} / \mathrm{mm}$, the $N$-domains gradually disconnect from each other and become significantly smaller within a field-amplitude range of about $0.2 \mathrm{~V} / \mathrm{mm}$. The image of this phase is given in Fig. 3. This phase will be referred to as the
$N_{D}{ }^{*}$-phase, where the subscript " $D$ " stands for "disconnected $\mathrm{N}$-domains". The striped texture melts at a field amplitude of $3.3 \mathrm{~V} / \mathrm{mm}$, and at the same time the $N$-domains melt and reform. There is no sign of macroscopic flow. This dynamical state is referred to as the $D_{s}$-state. A snapshot of this dynamical state is given in Fig. 3. The subscript " $s$ " stands for "slow", which is added because a long time (larger than about $5 \mathrm{~s}$ ) is required for melting and reforming of $\mathrm{N}$-domains close to the phase/state transition line. On increasing the field amplitude, the dynamics of melting and formation becomes faster, and remains essentially unchanged for field amplitudes above $4.2 \mathrm{~V} / \mathrm{mm}$, indicated by the dashed line. The dynamics of melting and reformation will be discussed in more detail in subsection IV C. The region in the phase/state diagram at higher field amplitudes where the time for melting and formation is relatively fast and essentially independent of the field amplitude is referred to as the $D_{f}$-state, where the subscript " $f$ " stands for "fast".

The above described phases and states are found to be independent of the way they are reached. For example, $N$-domains are formed from the $N^{*}$-phase on lowering the field amplitude. This shows that the state where $N$-domains are in coexistence with isotropic regions is a stable phase.

At high frequencies, larger than a few $\mathrm{kHz}$, the depolarized microscopy image is uniform, as shown in the left image in Fig. 4. As will be seen in subsection IV D, the rods are aligned along the direction of the electric field in this uniform phase. This phase is referred to as the $H$-phase, where " $H$ " stands for homeotropic. On decreasing the frequency for a given field amplitude of $4 \mathrm{~V} / \mathrm{mm}$, the $N^{*}$-phase is formed, where the striped texture is quite irregular and the pitch is very large (about 50-100 $\mu \mathrm{m}$ ), as can be seen from the middle image in Fig. 4. The pitch decreases

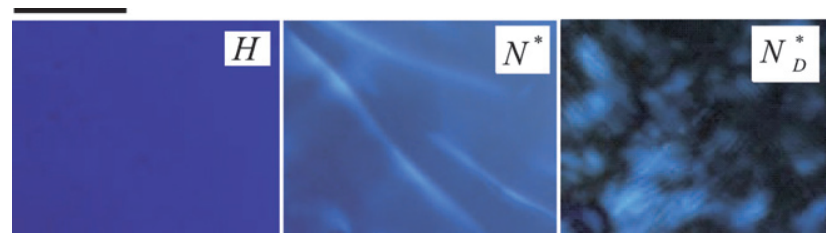

Fig. 4 Depolarized optical morphologies of the various phases at a medium field amplitude of $4 \mathrm{~V} / \mathrm{mm}$ on decreasing the frequency (for 2.8 $\mathrm{mg} / \mathrm{ml}$ ): the $H$-phase (at $4 \mathrm{kHz}$ ), high frequency $N^{*}$-phase (at $800 \mathrm{~Hz}$ ), and the $N_{D}{ }^{*}$-phase (at $200 \mathrm{~Hz}$ ). The scale bar is $200 \mu \mathrm{m}$, and the electric field is perpendicular to the field of view.
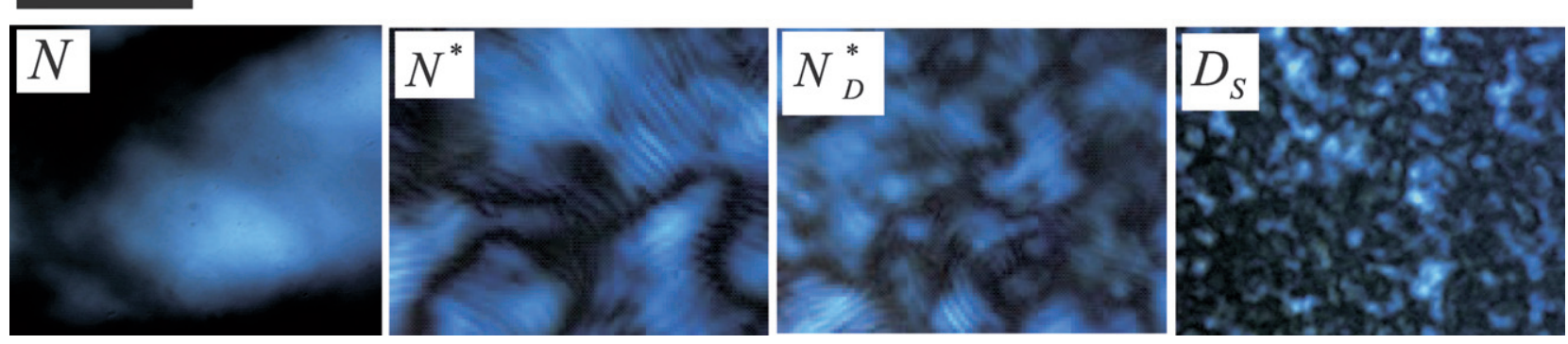

Fig. 3 Typical depolarized optical morphologies of the various phases at low frequency $(10 \mathrm{~Hz})$ on increasing the field amplitude, for a fd-concentration of $2.8 \mathrm{mg} / \mathrm{ml}$ : the $N$-phase, the stripe-textured $N^{*}$-phase, the $N_{D}{ }^{*}$-phase, and a snapshot of the dynamical $D_{s}$-state. The scale bar is $200 \mu \mathrm{m}$, and the electric field is perpendicular to the field of view. The field amplitudes are, from left to right, 1.0, 1.7, 2.7 and $3.4 \mathrm{~V} / \mathrm{mm}$. 
on subsequently decreasing the frequency. The $N_{D}{ }^{*}$-phase is entered on further lowering the frequency (see the right image in Fig. 4).

In Ref. [8] we denoted the $H$-phase as the isotropic $I$-phase. This was erroneously based on birefringence measurements on the $2.0 \mathrm{mg} / \mathrm{ml}$ sample at incident angles of only $\delta=10^{\circ}$ (see subsection IV D). The resulting phase shift is zero within experimental error, so that we erroneously concluded that this phase is isotropic. We now have definite proof, as discussed in subsection IV D, that this phase is indeed a homeotropic phase.

The same phases and states are found for both fd concentrations (see Fig. 2), with different locations of the transition lines. For the lower fd concentration of $2.0 \mathrm{mg} / \mathrm{ml}$, a "critical point" is found within the probed amplitude range (at $300 \mathrm{~Hz}$ and $4.5 \mathrm{~V} / \mathrm{mm}$ ), where several transition lines meet. In fact, one might refer to this point as a "triple or quadruple point", since several transition lines meet there. Detailed experiments on the nature of this "non-equilibrium critical point" have been performed. ${ }^{23}$ For the higher fd concentration of $2.8 \mathrm{mg} / \mathrm{ml}$, the "critical point" is located at higher frequency and higher field amplitude (see Fig. 2b).

We also determined the location of some of the transition lines discussed above for various fd concentrations at a fixed low frequency, which are given in Fig. 5. As can be seen, the field amplitudes where the $N$-to- $N^{*}$ and the $N_{D}{ }^{*}$-to- $D_{s}$ transitions occur rapidly decrease with decreasing fd concentration. Below an fd concentration of about $1.5 \mathrm{mg} / \mathrm{ml}$, an isotropic phase is observed in the absence of an electric field, with a weak degree of alignment when an electric field is applied (as discussed in subsection IV D). This phase is indicated in Fig. 5 by " $I$ ". Note that the field amplitude where the $I$-phase transition line is located is a very strong function of concentration. For this reason, the $D_{f}$-to- $I$ transition could only be observed at relatively high field amplitudes for a given fd concentration. Note that no new phase can be induced when the stable phase is isotropic in the absence of an electric field (this was verified up to field amplitudes of $10 \mathrm{~V} / \mathrm{mm}$ ).

\section{Characterization of transition lines, phases and states}

In this section, phases, states and transition lines are further characterized. It will be shown that the $N$-phase is a coexistence

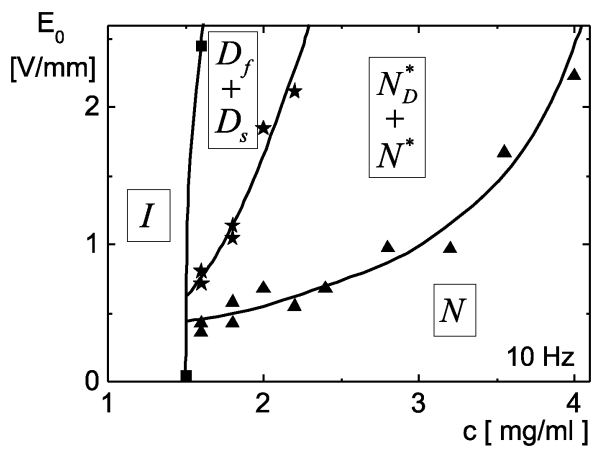

Fig. 5 The electric phase/state diagram as a function of fd-concentration at a fixed frequency of $10 \mathrm{~Hz}$. between nematic and isotropic regions. We will speculate on the nature of the striped texture, where there are strong indications that the striped texture corresponds to a chiral nematic. Pitch variations on approach of the $N$-to- $N^{*}$ transition line on lowering the field amplitude are measured. It will be shown that the microscopic dynamics exhibit a discontinuity at the $N_{D}{ }^{*}$-to- $D_{s}$ transition. The dynamics of melting and forming of $N$-domains in the dynamical $D_{s^{-}}$and $D_{f}$-states will be discussed. Finally, birefringence measurements on orientational order in the highfrequency uniform $H$-phase are presented.

\section{A. The $N$-, $N^{*}$ - and $N_{D}{ }^{*}$-phases}

In the existing literature, the nematic phase of fibrous virus suspensions (in the absence of an external field) is found to be chiral-nematic, ${ }^{24-27}$ as a result of the chiral structure of the core of fd-virus particles. These studies are all performed at relatively high ionic strengths (for analytical TRIS/HCl-buffer concentrations larger than about $5 \mathrm{mM}$ ). In the present study, a much lower analytical buffer concentration of $0.16 \mathrm{mM}$ is used. The corresponding much larger Debye length screens the chiral structure of the charged core of fd-virus particles to an extent that renders the nematic phase non-chiral in the absence of an external field.

A coexistence between a nematic and an isotropic phase in the absence of a field is confirmed by gentle centrifugation of a bulk phase (containing the $2.8 \mathrm{mg} / \mathrm{ml}$ fd suspension; see left image in Fig. 6a), in order to macroscopically phase separate the isotropic and nematic regions. After gentle centrifugation, we find two stable phases, an upper isotropic phase and a lower nematic phase, as shown in the right photo in Fig. 6a. The concentration of the isotropic phase is measured to be $1.33 \pm 0.10 \mathrm{mg} / \mathrm{ml}$, which is in accordance with the lower binodal concentration that we found in the phase diagram in Fig. 5. An isotropic-nematic coexistence is confirmed by taking images of the electric cell between crossed polarizers with varying orientation of both polarizers. Such images are shown in Fig. 6b. The solid line encloses an $N$-domain, which turns from bright to dark and to bright again on rotation of the polarizers by $90^{\circ}$. The dashed lines enclose areas that remain black, independent of the orientation of the crossed polarizers. These correspond to either isotropic regions or to $\mathrm{N}$-domains that happen to have their director perpendicular to the field of view.

For our low salt concentration of $0.16 \mathrm{mM}$, a non-chiral nematic is observed, while a striped pattern is only found at finite electric field amplitudes. By applying an electric field, the double layer is partly destroyed, possibly resulting in a reduction of the range of electrostatic interactions. Just as for the high-salt case, this would result in the formation of a chiral nematic. A microscopic verification of the chiral nature of the $N^{*}$-phase requires a separate study that is beyond the scope of the present paper.

The striped texture extends over regions that exceed the size of the $N$-domains, and it does not nucleate within the $N$-domains. The $N$-to- $N^{*}$ transition is therefore not related to a Frederick's transition corresponding to anchoring-orientational order at the boundaries of $\mathrm{N}$-domains. Furthermore, no isotropic regions were found, by taking images similar to those in Fig. 6 . 


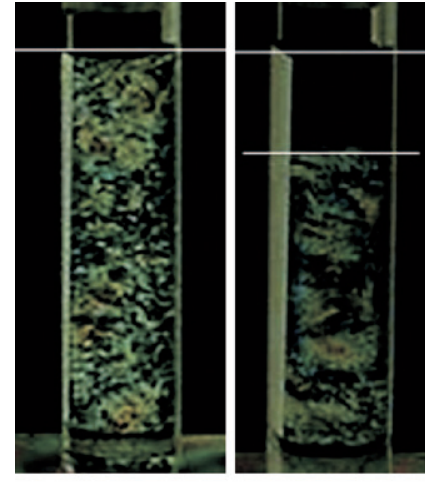

(a)

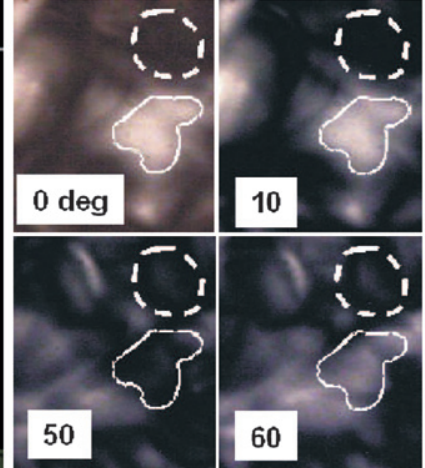

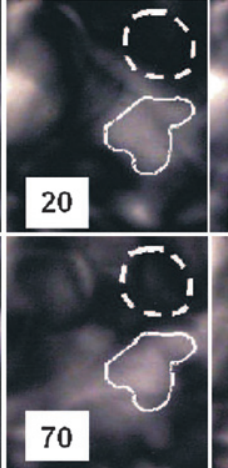

(b)

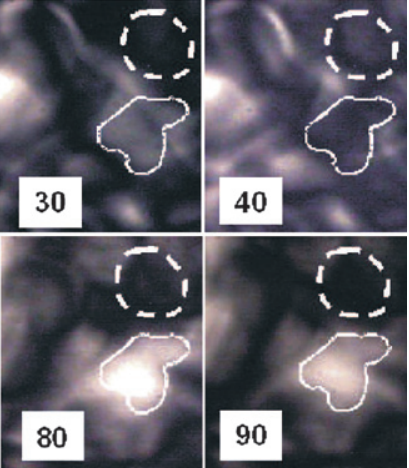

Fig. 6 (a) A bulk sample between crossed polarizers (left, before, and right, after centrifugation) where macroscopic phase separation has been achieved by gentle centrifugation. The black upper phase is isotropic and the lower phase exhibits the typical nematic texture. (b) Images of the $N$-phase on changing the angle of the crossed polarizers in steps of 10 degrees relative to the sample. The dotted areas are regions that remain black for all angles, while the solid line is an example of a region that contains an $N$-domain.

Variation of the optical pitch of the assumed chiral nematic in the $N^{*}$ - and $N_{D}{ }^{*}$-phase on increasing the field amplitude has been measured directly from depolarized images. ${ }^{8}$ The variation of the pitch and its apparent divergence on approach of the $N$-to- $N^{*}$-transition line on lowering the field amplitude, is illustrated in the images in Fig. 7. Since the pitch diverges at the $N$-to- $N^{*}$-transition line, this line may be referred to as a line of Lifshitz points. As discussed in Ref. [8], there is a strong variation in the measured pitch at low field amplitudes, which becomes smaller with increasing amplitudes. Just above the $N$-to- $N^{*}$ transition line (at $1.1 \mathrm{~V} / \mathrm{mm}$ ) the variation in the measured pitch ranges from about $12 \mu \mathrm{m}$ to $32 \mu \mathrm{m}$. The large spread at low field amplitudes can be interpreted as being the result of a random orientation of the director. The pitch levels off to about $10 \mu \mathrm{m}$ at high field amplitudes within the $N_{D}{ }^{*}$-phase.

\section{B. Microscopic dynamics at the $N_{D}{ }^{*}$-to- $D_{s}$ transition}

A vertically mounted Dynamic Light Scattering (DLS) setup is used to probe the dynamics of fd-virus particles on a microscopic length scale (of the order of a $\mu \mathrm{m}$ ). The principle set up is sketched in Fig. 8a. Due to scattering by the glass-water and glass-air interfaces, there is typically about $20 \%$ heterodyning contribution. It turned out that the use of a lens (with a focal length of $75 \mathrm{~mm}$ ) is necessary in order to eliminate contributions from particles close to the walls of the planar electrical cell. Without the lens, the long-time part of correlation functions is
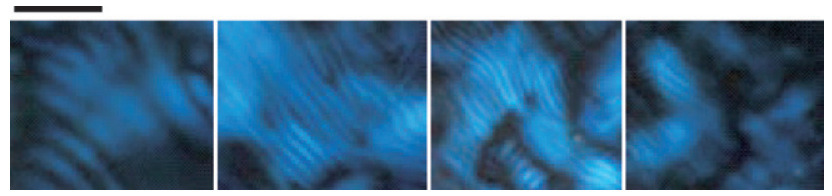

Fig. 7 Optical morphologies in the $N^{*}$ and $N_{D}{ }^{*}$ phase, showing the variation of the chiral pitch with field amplitude, at a fixed frequency of $10 \mathrm{~Hz}$, and an fd-concentration of $2.8 \mathrm{mg} / \mathrm{ml}$. The scale bar indicated above the left image is $200 \mu \mathrm{m}$. The field amplitudes are, from left to right, $1.1,1.5,1.9$ and $2.3 \mathrm{~V} / \mathrm{mm}$. affected through the scattering of particles close to the wall. As can be seen in Fig. 8b, the diffusion coefficients obtained from the vertical DLS set up (0) compare well with those obtained from a standard ALV set up ( $\square$ ). The accuracy of measured diffusion coefficients is about $10 \%$. In obtaining diffusion coefficients from measurements with the vertical DLS set up, the actual scattering vector $q$ is corrected for refraction at the cell interfaces.

The vertical DLS set up is used to probe the microscopic dynamics of rods around the $N_{D}{ }^{*}$-to- $D_{s}$ transition line, at $10 \mathrm{~Hz}$ for an fd concentration of $2.8 \mathrm{mg} / \mathrm{ml}$. Typical correlation functions are shown in Fig. 9. The interpretation of such correlation functions in terms of relaxation processes is not possible, since the sample is highly inhomogeneous and, within the $D_{s}$ state, melting and forming of $N$-domains occurs. The only purpose of the DLS measurements is to obtain a measure for the average "mobility" of fd-virus particles. As can be seen from Fig. 9, the correlation functions exhibit a two-stage decay. The slower mode is fitted to the stretched exponential form in
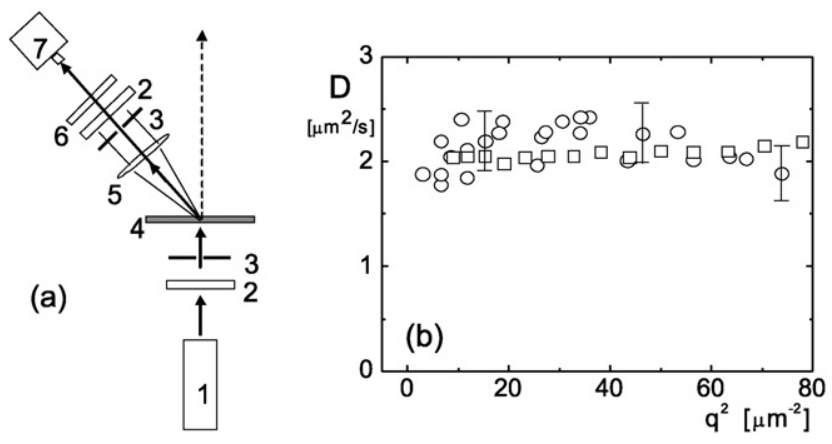

Fig. 8 (a) A schematic drawing of the vertical DLS setup: (1) $\mathrm{He}-\mathrm{Ne}$ laser, (2) polarizers, (3) pinholes (diameter of $500 \mu \mathrm{m}$ ), (4) sample, (5) achromatic lens (focal length $75 \mathrm{~mm}$ ), (6) bandpass filter $(632 \mathrm{~nm}),(7)$ mono-mode fiber and APD. (b) A comparison of the diffusion coefficient of a dilute dispersion of silica spheres from a standard ALV DLS set up $(\square)$ and our vertical DLS set up $(\bigcirc)$, where the sample is contained in the electrical cell without an electric field. 


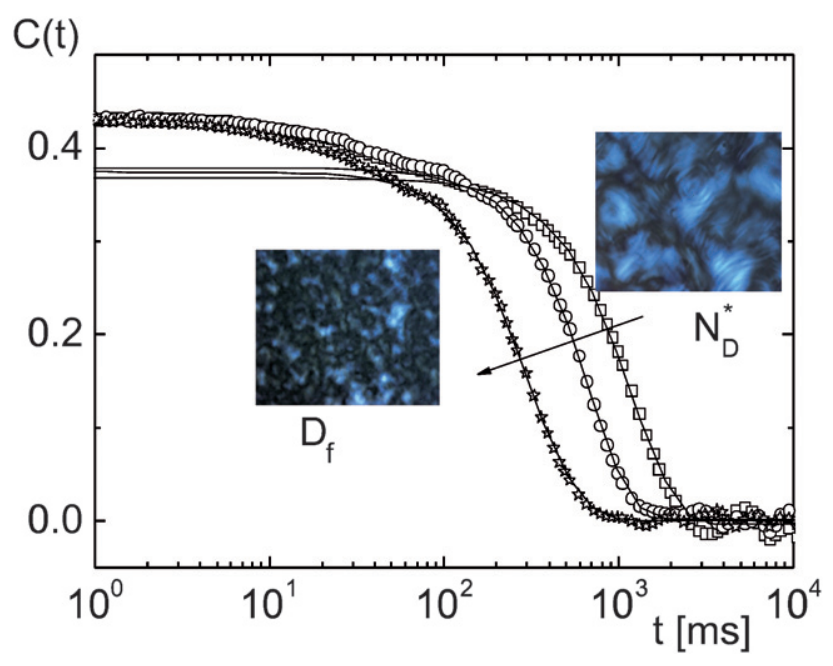

Fig. 9 Examples of DLS-correlation functions for a fd concentration of $2.8 \mathrm{mg} / \mathrm{ml}$, with increasing field amplitude: $N_{D}^{\star}$ ( $\square$, the right upper image), $D_{s}(\bigcirc)$ and $D_{f}(\star$, the left lower image). The solid lines are fits to the stretched exponential function for $g_{1}$ in eqn(2), with $B=0$, accounting for partial heterodyning. The scattering angle is fixed at $21^{\circ}$, which corresponds to a length scale of $1 \mu \mathrm{m}$.

eqn (2) for the electric-field correlation function $g_{1}$ (with $B=0$ ), including partial heterodyning. The decay time $\tau$ is taken as a measure for the mobility of the rods. The solid lines in Fig. 9a are fits to this form for $g_{1}$. The resulting values for the time constant $\tau$ as a function of the field amplitude exhibit a discontinuity at the $N_{D}{ }^{*}$-to- $D_{s}$-transition line. ${ }^{8}$ The microscopic dynamics of fd rods thus becomes faster in a discontinuous fashion when the dynamic state is entered. The time scale on which melting and forming of $N$-domains just inside the $D_{s}$-state occurs is an order of magnitude larger than the decay time of the corresponding DLS correlation function. This implies that the observed discontinuity in the microscopic dynamics is not connected to the dynamical features of the $D_{s}$-state, but is due to differences in microstructural order in the $N_{D}{ }^{*}$-phase and the $D_{s}$-state. It is possibly connected to the sudden disappearance of the striped texture on entering the $D_{s}$-state. Further investigations are needed to clarify the differences in microscopic ordering that lead to the sudden increase of the mobility of the rods.

\section{The dynamics of melting and formation of $\mathrm{N}$-domains in the $D_{s^{-}}$and $D_{f^{-}}$-states}

The dynamics of melting and formation of $\mathrm{N}$-domains are accelerated on increasing the field amplitude just above the $N_{D}{ }^{*}$ to- $D_{s}$ transition line. Time traces of the transmitted intensity through crossed polarizers, as detected by the CCD camera for a single pixel, are shown in Fig. 10a,b. As can be seen, the variations in the intensity trace within the $D_{f}$-state in Fig. 10a are much faster as compared to the $D_{s}$-state in Fig. 10b. A quantitative measure for the average time $\tau$ for $N$-domains to melt and form can be obtained from correlation functions of such time traces. The video-intensity correlation function $C_{\mathrm{V}}$ that is obtained from CCD images is defined as,
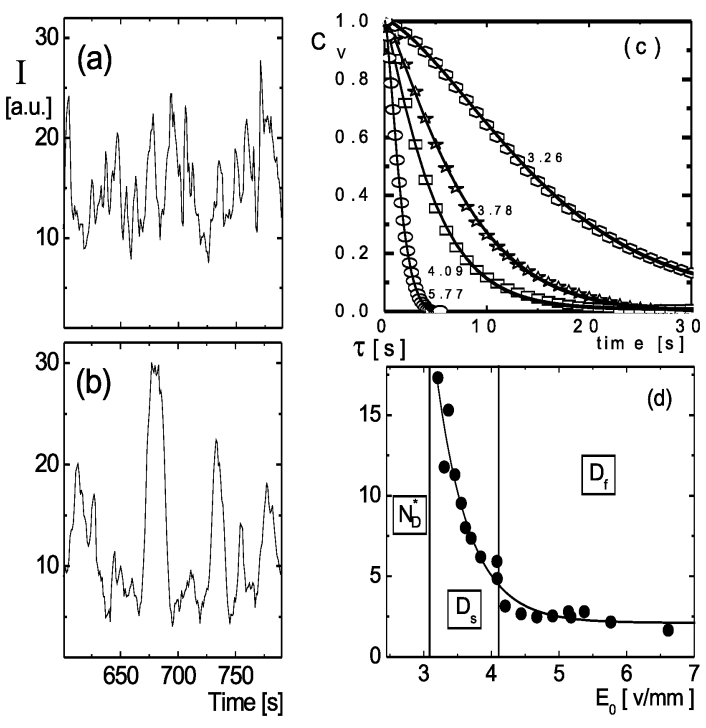

Fig. 10 Intensity time traces of a selected camera pixel in the dynamical state (a) $D_{f}$ and (b) $D_{s}$, showing the significantly faster dynamics in the $D_{f}$-state as compared to the $D_{s}$-state. (c) Video-correlation functions $C_{V}$ of intensity traces for four field amplitudes (the numbers in the figure indicate the applied field amplitude in $\mathrm{V} / \mathrm{mm}$ ). The solid lines are fits to a stretched exponential (see eqn(2)). (d) The decay times as obtained from fits of video-correlation functions to a stretched exponential as a function of the field amplitude. The different phases and states are indicated.

$$
C_{V}(t)=\frac{<[I(t)-<I(t)>][I(0)-<I(0)>]>}{<[I(0)-<I(0)>]^{2}>}
$$

where $I(t)$ is the intensity of a pixel at time $t$, while the brackets $<\cdots\rangle$ indicate averaging over all pixels. Examples of such correlation functions are shown in Fig. 10c, for various field amplitudes. The solid lines in this figure are fits to the stretched exponential function,

$$
F(t)=B+A \exp \left\{-(t / \tau)^{\beta}\right\}
$$

where $B, A, \beta$ and $\tau$ are fitting parameters. The time constant $\tau$ is a measure for time with which $N$-domains melt and form. The time constant is plotted in Fig. 10d as a function of the field amplitude, where the $N_{D}{ }^{*}$-to- $D_{s}$ phase transition is indicated, as well as the $D_{s}$-to- $D_{f}$ transition line. As can be seen, $\tau$ diverges on approach of the $N_{D}{ }^{*}$-to- $D_{s}$ phase transition on lowering the field amplitude. The transition from the $D_{s^{-}}$to the $D_{f}$-state is defined as the point where the sharp decrease of $\tau$ with increasing field amplitude ceases to occur, and the dynamics becomes essentially independent of the field amplitude.

\section{Orientational order in the $\boldsymbol{H}$-phase}

In view of the uniformity of the left image in Fig. 4, there are two possible rod orientations in the high frequency $H$-phase. One possible orientation is homeotropic, where rods are aligned perpendicular to the electrodes, that is, along the electric field. The other is planar orientation, where rods are aligned perpendicular to the field, with an isotropic distribution within the plane parallel to the electrodes. 

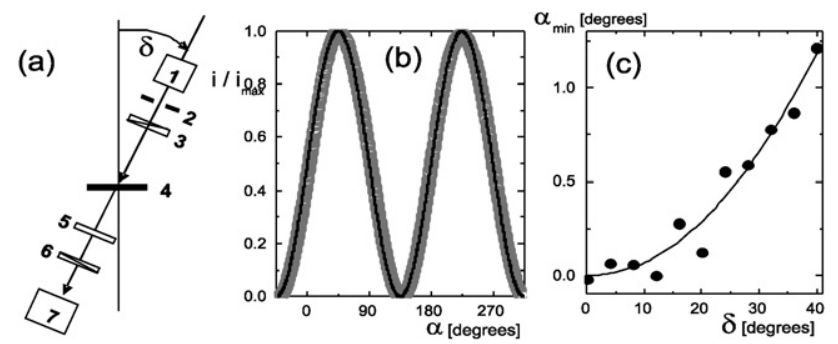

Fig. 11 (a) The experimental scheme of the electric birefringence set up: (1) a diode laser (wavelength $663 \mathrm{~nm}$ ), (2) pinhole, (3) Glan-Thompson polarizer, (4) electrical sample cell, (5) $\lambda / 4$-platelet, (6) Glan-Thompson polarizer mounted on a $360^{\circ}$ motorized stage, and (7) the detector. The optical train makes an angle $\delta$ with the normal to the electrical cell. (b) The transmitted light intensity $i$ (normalized to the maximum intensity $\left.i_{\max }\right)$ as a function of the analyzer angle $\alpha$, for an angle of incidence of $\delta=$ $20^{\circ}$. The solid line is the function in eqn(3) with $\Delta \Phi=0$. (c) The analyzer angle $\alpha_{\min }$ where the minimum intensity is found as a function of the angle of incidence $\delta$, where the electrical cell is filled with water. The "apparent birefringence" is due to refraction at the glass-air and glasswater interfaces.

Orientational order in the high-frequency $H$-phase is probed with the vertical birefringence set up as depicted in Fig. 11a. A laser beam passes through a Glan-Thompson polarizer, which is oriented at $45^{\circ}$ with respect to the direction that is perpendicular to the plane in which the entire optical train can be rotated. Below the horizontally placed electrical sample cell, a $\lambda / 4$-platelet is positioned with one of its optical axes parallel to that of the polarizer. The transmitted intensity is measured as a function of the angle $\alpha$ of the orientation of a second Glan-Thompson polarizer. When a birefringent sample leads to a phase shift $\Delta \Phi$, the transmitted intensity $i$ varies with the angle $\alpha$ of the analyzer as,

$$
i=1 / 2 i_{\max }[1+\sin (2 \alpha+\Delta \Phi)]
$$

where $i_{\max }$ is the maximum transmitted intensity. This variation of the intensity is verified in Fig. 11b, in the absence of the sample cell.

In order to be able to measure birefringence due to possible homeotropic or planar alignment, it is necessary to rotate the entire optical train. The laser beam then makes an angle $\delta$ with the normal to the flat electrodes of the sample cell (see Fig. 11a). Measurements on the $H$-phase at normal incidence (with $\delta=0$ ) show that there is no birefringence within directions parallel to the electrodes, in accord with the uniform intensity distribution under crossed polarizers in the left image in Fig. 4. Due to refraction at the water-glass and glass-air interfaces of the sample cell, an "apparent birefringence" is measured, which increases with increasing angle of incidence $\delta$. The analyzer angle $\alpha_{\min }$ where the measured transmitted intensity through the sample cell filled with water attains its minimum value is plotted in Fig. 11c against the angle of incidence. The shift of $\alpha_{\min }$ due to the cell interfaces is seen to be less than about $0.2^{\circ}$ for $\delta$ not larger than about $20^{\circ}$. This is sufficiently small to perform accurate birefringence measurements at $\delta=20^{\circ}$. From the phase shift $\Delta \Phi$, the "measured birefringence" $\Delta n_{\text {meas }}$ can be calculated from,

$$
\Delta n_{\text {meas }}=\frac{1}{2 \pi} \frac{\lambda}{d} \Delta \Phi
$$

where $\lambda=663 \mathrm{~nm}$ is the laser wavelength in vacuum and $d=d_{0} / \sqrt{1-n^{-2} \sin ^{2}(\delta)}$ is the optical path length, with $d_{0}$ the distance between the two electrodes and $n$ the refractive index of the suspension. Note that the sign of the measured phase shift $\Delta \phi$ depends on the orientation of the fast axis of the $\lambda / 4$-platelet as well as the direction of alignment of the rods. As shown in appendix A, the difference $\Delta n$ between the refractive indices in the sample along its optical axis perpendicular and parallel to the electrodes is related to the measured birefringence as,

$$
\Delta n=\Delta n_{\text {meas }} / \sin ^{2}(\delta)
$$

provided that $\Delta n$ is small compared to the mean refractive index.

In order to establish whether a positive or negative phase shift corresponds to homeotropic or planar orientation of the rods, measurements are performed on a shear-aligned suspension of a high nematic concentration $(6.4 \mathrm{mg} / \mathrm{ml})$. The rods are shearaligned by gently moving the upper plate of the sample cell relative to the lower plate. The direction of shear alignment of the rods is parallel to the electrodes and along the direction of shearing. The birefringent intensity is measured, without an electric field, for two orientations of the sample cell under normal incidence (with $\delta=0$ ): one where the direction of orientation of $\mathrm{fd}$ is parallel to the plane in which the detector can move, which is the plane of the paper in the schematic drawing in Fig. 11a, and one perpendicular to that plane. The measured intensities are shown in Fig. 12 for these two cases, as well as for the pure buffer. As expected, the sign of $\Delta n_{\text {meas }}$ changes on rotating the sample over $90^{\circ}$, but the magnitude of the phase shift remains the same. From the thus observed sign of the shift of the analyzer angle $\alpha_{\min }$ where the intensity attains a minimum value, it follows that a negative angular shift corresponds to homeotropic alignment, while a positive shift corresponds to planar orientation. Details can be found in appendix A.

In Fig. 13a,b, the analyzer angle $\Delta \alpha$ relative to that of pure buffer for the $H$-phase is shown for two fd concentrations 2.0 and $2.8 \mathrm{mg} / \mathrm{ml}$, respectively, where at a fixed frequency the field amplitude is either small (just inside the $H$-phase) or large. First

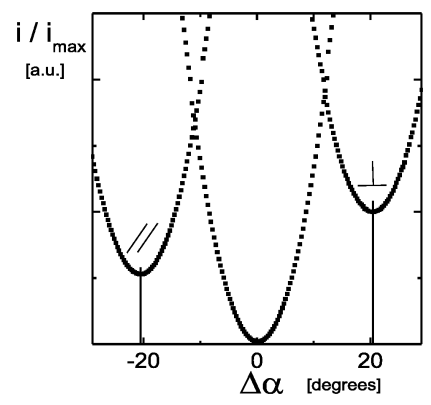

Fig. 12 The measured transmitted intensity of a pre-sheared sample with a fd concentration of $6.4 \mathrm{mg} / \mathrm{ml}$, which is in the one-phase nematic region, without an electric field. For the curve marked $\|$, the direction of alignment of the rods is within the plane in which the detector can move (on changing $\delta$ ). For the curve marked $\perp$, the rods are aligned in the direction perpendicular to that plane. The middle curve is pure buffer. 

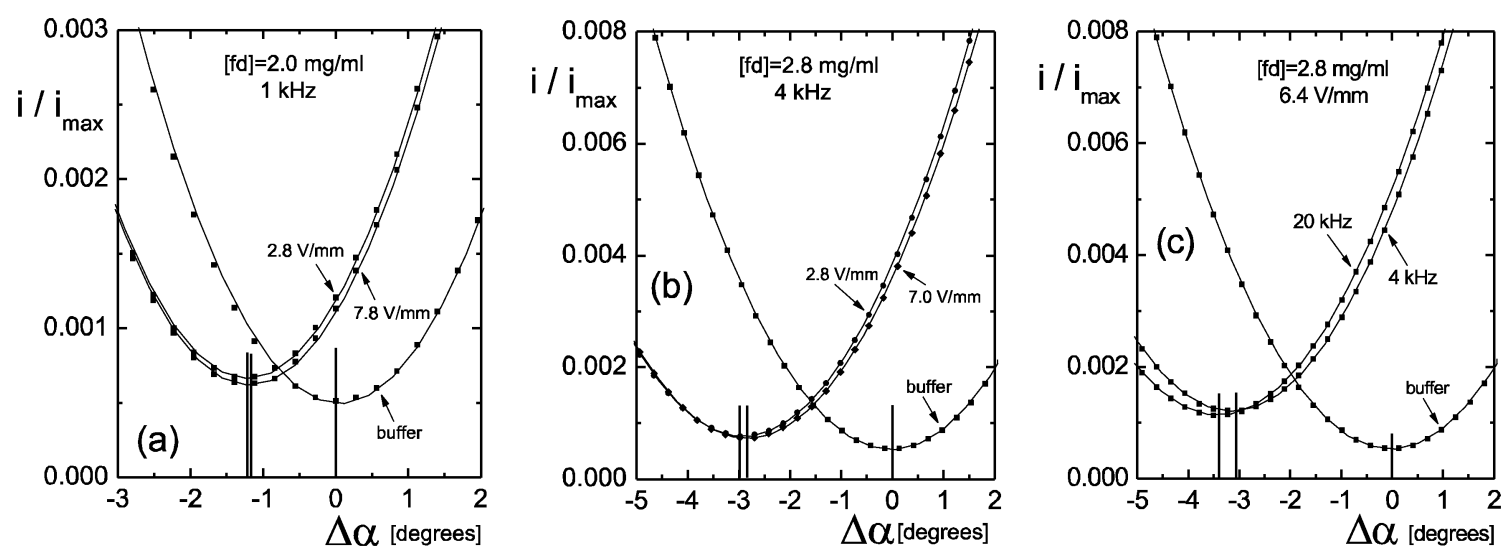

Fig. 13 (a) Birefringence measurements at $\delta=20^{\circ}$ incidence in the $H$-state at $1 \mathrm{kHz}$ for an fd concentration of $2.0 \mathrm{mg} / \mathrm{ml}$. Plotted is the transmitted intensity $i$, normalized to the maximum transmitted intensity $i_{\max }$, as a function of the analyzer angle $\Delta \alpha$ relative to that of the angle where the buffer attains its minimum transmitted intensity. The solid curves are fits to a second order polynomial, from which the location of minimum is calculated. The curve marked as "buffer" is taken for the cell filled with pure buffer, without fd, while the other curves are taken with fd at the indicated applied voltages. (b) The same as in (a) but now for the fd concentration of $2.8 \mathrm{mg} / \mathrm{ml}$, at $4 \mathrm{kHz}$. (c) The same as in (b), where now the field amplitude is fixed at $6.4 \mathrm{~V} / \mathrm{mm}$ and the frequency is varied.

of all, the angular shift is negative in all cases. As discussed above, this implies that the rods align along the applied electric field. Secondly, the shift is essentially independent of the applied field amplitude. Moreover, the degree of alignment is independent of the frequency, as can be seen from Fig. 13c. No significant differences of the degree of alignment have been found on increasing the frequency from $4 \mathrm{kHz}$ up to $20 \mathrm{kHz}$ for the $2.8 \mathrm{mg} /$ $\mathrm{ml}$ and a field amplitude of $6.4 \mathrm{~V} / \mathrm{mm}$. These measurements imply that within the probed range of field amplitudes and frequencies in the phase diagrams in Fig. 2, the degree of homeotropic alignment is essentially constant. There is, however, a pronounced fd-concentration dependence, as can be seen by comparing Fig. 13a and b. The angular shift for 2.0 and $2.8 \mathrm{mg} /$ $\mathrm{ml}$ in the analyzer angle $\alpha$ where the intensity attains its minimum vales is $-1.2^{\circ}$ and $-2.9^{\circ}$, respectively (with an experimental error of about $0.2^{\circ}$ ). The orientational order parameter $S$ can be obtained from,

$$
S=|\Delta n| / \Delta n_{\max }
$$

where $\Delta n_{\max }=[3.8 \pm 0.3] \times 10^{-5} \times c$, with $c$ the fd concentration in units of $\mathrm{mg} / \mathrm{ml} .^{28}$ The order parameter in the $H$-phase as

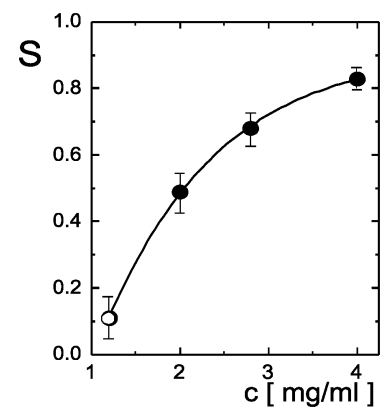

Fig. 14 The orientational order parameter in the $H$-phase as a function of fd-concentration. The measured order parameter at the lowest concentration (the open circle) is just beyond experimental error, and relates to the $I$-phase in the phase diagram in Fig. 5. a function of concentration is given in Fig. 14. As can be seen from Fig. 14, there is a strong increase of the degree of homeotropic alignment with increasing fd concentration. For the lower fd concentration of $1.2 \mathrm{mg} / \mathrm{ml}$, where there is only a single uniform phase, we observed a very small order parameter, just within experimental error, that is essentially independent of the field amplitude and the frequency in the probed range of 0.1-10 $\mathrm{V} / \mathrm{mm}$ and up to $20 \mathrm{kHz}$, similar to the $H$-phase. Because of the very small degree of orientational order in this phase, we preferred to refer to it as the isotropic phase $I$.

\section{Electrode polarization}

When a time-independent electric field is employed, double layers will be present at both electrodes which screen the electric field over a distance of the order of the Debye length. The electric field in the bulk of the solution is therefore zero, so that the double layers act as "perfect insulators". The charges within the double layers compensate the charges that are externally applied to the electrodes. Due to the finite diffusivity of the salt ions, the double layer at the electrodes will not fully develop when an oscillatory external field is applied. The electric field in the bulk is now non-zero. Due to the charges near the electrodes within the partly developed double layers, however, the electric field in the bulk is not equal to the applied field. This screening of the electric field by electrode double layers is commonly referred to as "electrode polarization". At very high frequencies, where ions are not able to respond to electrical forces, the electric field in the bulk becomes equal to the applied field, and renders electrode polarization insignificant.

We consider the geometry that is used in our experiments, where two flat electrodes confine the suspensions. The externally applied electric field $E^{\text {ext }}$ to the electrodes is given by,

$$
E^{e x t}=E_{0} \cos \{\omega t\}
$$

where $E_{0}$ is the applied field amplitude and $\omega$ the frequency. Due to electrode polarization, the actual electric field $E^{\text {bulk }}$ in the bulk of the suspension, in the absence of the colloids, has an amplitude 
$E$ that is different from the applied amplitude $E_{0}$ and is phase shifted by an angle $\varphi$ as compared to the applied field,

$$
E^{\text {bulk }}=E \cos \{\omega t+\varphi\}
$$

The ratio of the field amplitude $E$ in the bulk and the applied amplitude $E_{0}$ defines the "attenuation factor" $\gamma$,

$$
E=\gamma E_{0}
$$

Both $\varphi$ and $\gamma$ depend on the frequency, the Debye screening length, the cell thickness and the diffusion coefficient of the small ions.

In any theory for the polarization of double layers of colloids, the input is the electric field strength experienced by the colloids, that is, the field strength $E^{\text {bulk }}$ in the bulk of the solution. In an experiment, however, the input is the applied field $E^{\text {ext }}$. Comparing theory and experiment thus requires an explicit expression for the attenuation factor $\gamma$. For a future comparison of our phase diagrams in Fig. 2 to theory, the applied field strength $E_{0}$ should therefore be corrected for electrode polarization by multiplication with the attenuation factor $\gamma$ in eqn (9) in order to obtain the phase diagram in terms of the field that is experienced by the colloidal particles.

In recent publications ${ }^{29,30}$ partly numerical solutions of the standard electro kinetic equations are presented in order to describe electrode polarization. No fully analytical expressions for ion densities and the electric potential in terms of the frequency, the Debye length, the cell thickness and the diffusion coefficient of small ions have been reported so far. Corrections for electrode polarization up to now therefore rely on numerical procedures. In the present section we shall derive a very simple expression for the attenuation factor, which requires no numerical procedures to correct data for electrode polarization. The Debye-Hückel approximation that we will make is shown to be valid for the typical values of the applied external field amplitudes. The point of departure is the same standard electro-kinetic equations for the ion concentrations and electric potential as used in the above mentioned references. For completeness, and to clearly state the approximations, the derivation of these wellknown equations (see, for example, ${ }^{11-14}$ ) is briefly discussed in subsection V A. The solution of the equations of motion, and the resulting expression for the attenuation factor and the phase shift will be presented in subsection V B. Mathematical details are given in appendix $\mathbf{B}$.

\section{A. The basic equations}

The ions experience a direct force $\mathbf{F}_{ \pm}$which sets them into motion with a velocity equal to $\mathbf{v}_{ \pm}=\mathbf{F}_{ \pm} / \zeta$, where $\zeta$ is the solvent friction coefficient of the ions. Note that for the two-plate geometry, where the applied field is perpendicular to the electrodes, there is no contribution to the ion velocities due to electro-osmotic flow. Electric body forces are now compensated by gradients in pressure. The direct force $\mathbf{F}_{ \pm}$has two contributions. First of all, there is a force due to the local electric field, resulting from the external field as well as charges within the electric double layers. This force on the \pm -ions is equal to $\mp e \partial \Phi(z, t) / \partial z$, where $e>0$ is the elementary charge and $\Phi$ is the local electric potential. Here, $z$ is the distance from the mid-plane between the two electrodes, where $z$ varies from $-L / 2$ to $+L / 2$, with $L$ the distance between the two electrodes. Secondly, there is the Brownian force $\mathbf{F}^{B r}{ }_{ \pm}=$ $-k_{B} T \partial \ln \left\{\rho_{ \pm}(z, t)\right\} / \partial z$, where $\rho_{ \pm}$is the local number density of ions, $k_{B}$ is Boltzmann's constant and $T$ the temperature. The Brownian force describes the diffusive motion induced by concentration gradients. Hence,

$$
\mathbf{F}_{ \pm}(z, t)=-k_{B} T \frac{\partial}{\partial z} \ln \left\{\rho_{ \pm}(z, t)\right\} \mp e \frac{\partial}{\partial z} \Phi(z, t)
$$

Substitution into the conservation equation $\partial \rho_{ \pm}(z, t) / \partial t=$ $-\partial\left[\rho_{ \pm} \mathbf{v}_{ \pm}(z, t)\right] / \partial z$ for the concentration thus leads to the wellknown equations of motion (we do not denote position and time dependence explicitly for brevity),

$$
\frac{\partial \rho_{ \pm}}{\partial t}=D \frac{\partial^{2}}{\partial z^{2}} \rho_{ \pm} \pm D \beta e \frac{\partial}{\partial z}\left[\rho_{ \pm} \frac{\partial}{\partial z} \Phi\right]
$$

where $\beta=1 / k_{B} T$ and $D=k_{B} T / \zeta$ is the Einstein diffusion coefficient of the ions.

We will limit ourselves to small electrostatic potentials. Since screening is entirely due to variation of ion concentrations close to the electrodes, within a region extending over distances equal to or less than the Debye length, the assumption is that the variation $\Delta \Phi$ of the potential over this distance away from the electrodes is small in the sense that $\beta e \Delta \Phi$ is small. Accounting for the significant dielectric screening of the applied potential in case of aqueous solvents, this condition is satisfied even for rather large applied potentials, as will be verified later experimentally (see subsection V C). Within this Debye-Hückel approximation, the excess density $\Delta \rho_{ \pm} \equiv \rho_{ \pm}-\bar{\rho}$, with $\bar{\rho}$ the overall density of ions, is proportional to the potential. Linearization of eqn(11) with respect to $\Delta \rho_{ \pm}$and $\Phi$ gives,

$$
\frac{\partial \Delta \rho_{ \pm}}{\partial t}=D \frac{\partial^{2}}{\partial z^{2}} \Delta \rho_{ \pm} \mp D \frac{1}{2} \kappa^{2}\left[\Delta \rho_{+}-\Delta \rho_{-}\right]
$$

where $\kappa=\sqrt{2 \beta e^{2} \bar{\rho} / \varepsilon}$ is the inverse Debye screening length. This equation of motion is the one-dimensional form of a wellknown electro-kinetic equation in the absence of electro-osmotic flow, which plays no role in the present plate geometry (see, for example, $\left.{ }^{11-14}\right)$.

The boundary condition for the potential between the electrodes reads,

$$
\Phi(z=1 / 2 L, t)-\Phi(z=-1 / 2 L, t)=E_{0} L \cos \{\omega t\}
$$

The second boundary condition specifies that ions can not penetrate the electrodes, that is, the ion fluxes $\rho_{ \pm} \mathrm{v}_{ \pm}$must vanish at the electrodes. Since ion velocities are proportional to the force in eqn (10), this implies that,

$$
\begin{aligned}
& \frac{\partial}{\partial z}\left[-\Delta \rho_{ \pm}(z, t) \mp \varepsilon \kappa^{2} \Phi(z, t)\right]=0, \\
& \text { for } z= \pm \frac{1}{2} \mathrm{~L}, \text { and all } t>0
\end{aligned}
$$

where the linearization discussed above has been invoked. 
For 1-1 electrolytes, the above equations can be used to derive equations of motion and boundary conditions for the free charge density $\rho=e\left[\Delta \rho_{+}-\Delta \rho_{-}\right]$due to differences in ion concentrations. Subtraction of the equation of motion (12) for $\Delta \rho_{+}$and $\Delta \rho_{-}$leads to a single equation of motion for the free charge density,

$$
\frac{\partial \rho}{\partial t}=D\left[\frac{\partial^{2}}{\partial z^{2}}-\kappa^{2}\right] \rho
$$

This equation of motion can be traced back to Ferry, ${ }^{31}$ who used it to describe the capacitance of a single double layer at a flat interface. The potential is connected to the free charge density through the Poisson equation,

$$
\frac{\partial^{2}}{\partial z^{2}} \Phi=-\frac{\rho}{\varepsilon}
$$

Subtraction of the boundary condition for $\Delta \rho_{+}$from that of $\Delta \rho_{-}$leads to,

$$
\frac{\partial}{\partial z}\left[\rho+\varepsilon \kappa^{2} \Phi\right]=0, \text { for } z= \pm \frac{1}{2} \mathrm{~L}, \text { and all } t>0
$$

The set $(13,15,16,17)$ of equations and boundary conditions describe electrode polarization within the Debye-Hückel approximation for 1-1 electrolytes.

The overall density of ions, in the case of a fd-suspension, is the concentration outside the double layers of the fd-viruses. This is the concentration that should be used to calculate the Debye length, and is equal to the concentration in the osmotic reservoir that is used in establishing osmotic equilibrium prior to the experiments. Even if the total amount of ions that dissociate from the fd surfaces exceeds the reservoir concentration, ion exchange in establishing osmotic equilibrium ensures an overall ion concentration that is set by the ion concentration in the osmotic reservoir. The number of ions that dissociate from the fd surfaces is rather limited, since about 80 to 90 percent of the bare charge is neutralized by condensed ions.

\section{B. The attenuation factor and phase shift}

Although the differential eqn (15), (16) have a quite simple appearance, their solution is not easily found and turns out to be quite complicated. The solution of these equations for the potential and the free charge density is constructed in the appendix B. As shown there, the in-phase and out-phase response functions for the electric field strength can be written as additive contributions $P^{\prime}{ }_{\text {bulk }}$ and $P^{\prime \prime}$ bulk that describe the electric field within the double layers near the electrodes, and contributions $P^{\prime}$ bulk and $P^{\prime \prime}$ bulk that describe the field within the bulk, away from the electrodes. Explicit expressions for both contributions are given in appendix $\mathrm{B}$. The contributions $P_{\mathrm{d} l}^{\prime}$ and $P^{\prime \prime}$ dl decay exponentially fast away from the electrodes over a distance that is at most equal to the Debye length. The electric field in the bulk of the solution, away from the electrodes, is thus written as,

$$
E^{b u l k}(z, t)=E_{0}\left[P_{b u l k}^{\prime}(z) \cos (\omega t)+P_{b u l k}^{\prime \prime}(z) \sin (\omega t)\right]
$$

The bulk response functions are given by,

$$
\begin{aligned}
& P_{b u l k}^{\prime}=1-\frac{2}{\varepsilon \kappa^{2} L\left(1+\Lambda^{2}\right)}[A(r-\Lambda s)+B(s+\Lambda r)] \\
& P_{b u l k}^{\prime \prime}=-\frac{2}{\varepsilon \kappa^{2} L\left(1+\Lambda^{2}\right)}[A(s+\Lambda r)-B(r-\Lambda s)]
\end{aligned}
$$

where $\Lambda=\omega / D \kappa^{2}$ is a dimensionless frequency, while explicit expressions for the constants $A, B, r$ and $s$ are given in eqn(37)-(39) in appendix B. For zero frequency, the bulk response functions vanish, that is, the electric field in the bulk solution is completely screened by the double layers at the electrodes. For very large frequencies, $P^{\prime}$ bulk tends to unity while $P^{\prime \prime}$ bulk vanishes, that is, the electric field in the bulk of the solution is now equal to the applied field. The double layers at the electrodes have no time to develop for these high frequencies, and therefore do not screen the applied electric field.

In experiments, the Debye length is very much smaller than the distance $L$ between the electrodes. Only the leading asymptotic form of the response functions in eqn (19) for large values of $\kappa L$ is therefore of interest. This leading order expansion is discussed in appendix B, leading to,

$$
\begin{aligned}
& P_{\text {bulk }}^{\prime}=\Omega^{2} /\left(4+\Omega^{2}\right), \\
& P_{\text {bulk }}^{\prime \prime}=-2 \Omega /\left(4+\Omega^{2}\right)
\end{aligned}
$$

where the dimensionless frequency $\Omega$ is defined as,

$$
\Omega=\omega L / D \kappa
$$

A similar expression for the relevant frequency has been derived in Ref. [30]. The physical meaning of this dimensionless frequency is as follows. Suppose that the distance $L$ between the electrodes is increased by a factor of two. In order to retain the same bulk electric field strength, the total potential difference between the electrodes, including the effects of ion-polarization charges, should be doubled. This requires a doubling of both the externally applied charges to the electrodes as well as the ionpolarization charges. Doubling the polarization charges requires typically twice as long. The frequency should therefore be half of the original frequency to retain the same bulk electric field strength. This explains why the frequency in the dimensionless group $\Omega$ in eqn (21) scales like $\omega \times L$. This feature renders electrode polarization dependent on the distance between the two plates, a fact that is used in electrophoresis experiments to measure essentially zero-frequency electrophoretic velocities using large electric cells.

Comparing the expressions (8) and (18) for the bulk electric field, the attenuation factor is found to be equal to,

$$
\gamma=\sqrt{\left(P_{\text {bulk }}^{\prime}\right)^{2}+\left(P_{\text {bulk }}^{\prime \prime}\right)^{2}}=\Omega / \sqrt{4+\Omega^{2}}
$$

while the phase shift between the applied field and the bulk electric field is given by,

$$
\varphi=\arctan \left(-P_{b u l k}^{\prime \prime} / P_{b u l k}^{\prime}\right)=\arctan (2 / \Omega)
$$

The bulk response functions for the electric field, the attenuation factor and the phase angle are plotted in Fig. 15). Note that the attenuation is significantly below unity for $\Omega<10$. This 

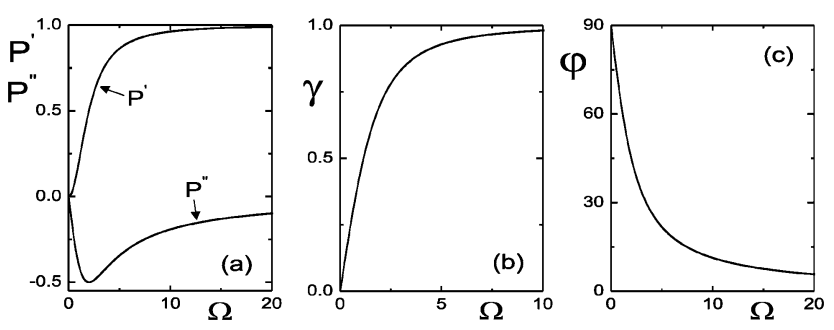

Fig. 15 (a) The bulk response functions $P^{\prime}$ and $P^{\prime \prime}$, (b) the attenuation factor $\gamma$ and (c) the phase angle (in degrees) as functions of the dimensionless frequency $\Omega$.

implies that electrode polarization becomes significant for frequencies below $10 \mathrm{DK} / L$.

\section{An experimental test of the theory and corrected phase diagrams}

The above theory for electrode polarization can be tested experimentally as follows. The value of electric field strength $E_{t r}{ }^{\text {bulk }}$ in the bulk of the suspension where a phase/state transition occurs is set by the response of the colloidal suspension, and is independent of the thickness of the cell. According to eqn(22) for the attenuation factor, the corresponding applied field amplitude $E_{0}$ is related to $E_{t r}{ }^{\text {bulk }}$ as,

$$
E_{0}=E_{t r}^{\text {bulk }} / \gamma=E_{\text {tr }}^{\text {bulk }} \sqrt{4+\Omega^{2}} / \Omega
$$

The applied field depends on the gap width $L$ of the electrical cell through the $L$-dependence of the dimensionless frequency $\Omega$ in eqn (21). The theory for electrode polarization can thus be tested by measuring the applied field strength that is necessary to induce a phase/state transition for several gap widths. The gapwidth dependence of the applied field can then be fitted to eqn (24) with two fitting parameters: $E_{t r}{ }^{\text {bulk }}$ and the diffusion coefficient $D$ of the small ions (which should be in the range of 1 to $3 \times 10^{-9} \mathrm{~m}^{2} / \mathrm{s}$ ). Probing several transitions, $E_{t r}{ }^{\text {bulk }}$ is an independent fitting parameter for each transition, while $D$ is a global fitting parameter that must be the same for all measurements. The externally applied transition field amplitudes $E_{0}$ for the transitions $N$-to- $N^{*}(\square)$ and $N_{D}{ }^{*}$-to- $D_{s}(\bigcirc)$ as a function of the cell-thickness $L$, for an fd concentration of $2.0 \mathrm{mg} / \mathrm{ml}$, is shown in Fig. 16. The solid lines are fits to eqn (24). The value for $D$ is found to equal to $2.1 \times 10^{-9} \mathrm{~m}^{2} / \mathrm{s}$ for both transition lines, which is perfectly within the expected range of literature values. The two transition field strengths are equal to 0.23 and $0.90 \mathrm{~V} / \mathrm{mm}$, respectively. Notice that these transition field strengths are yet to be corrected for dielectric polarization of the electrode-water interface. Since the static, relative dielectric constant of ITO (the electrode material) is approximately 7.5 and that of water is 78 , the actual transition field amplitudes are a factor $7.5 / 78 \approx 0.096$ smaller. Note that the applied field amplitudes corrected for dielectric polarization in Fig. 16 varies from about 50 to $300 \mathrm{mV}$, which is beyond the Debye-Hückel limiting value of about $25 \mathrm{mV}$. However, since inhomogeneities in free charge density only exist within the double layers, the theory is valid whenever the potential drop over the double layers is small compared to $25 \mathrm{mV}$. Since the Debye length is of the

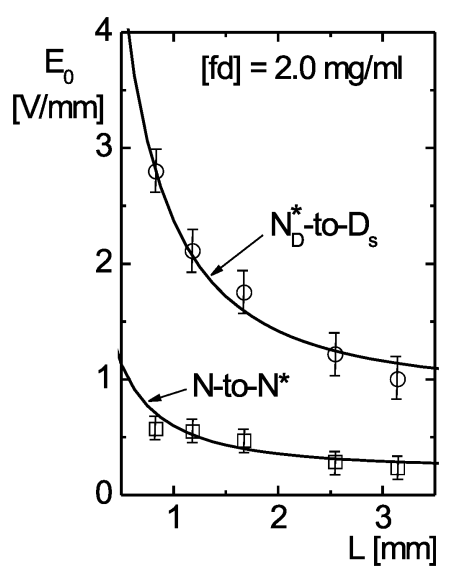

Fig. 16 The externally applied transition field amplitudes $E_{0}$ for the transitions $N$-to- $N^{*}(\square)$ and $N_{D}{ }^{*}$-to- $D_{s}(\bigcirc)$ as a function of the cellthickness $L$, for an fd concentration of $2.0 \mathrm{mg} / \mathrm{ml}$. The solid lines are fits to the theory (see eqn (24)).

order of a few $\mathrm{nm}$, it follows that the theory can be applied to seemingly quite high applied voltages, far above the maximum applied voltage of about $14 \mathrm{~V}$ in the present experiments. The bulk field amplitude $E=0.096 \times \gamma \times E_{0}$ is the field amplitude that is experienced by the colloids in the bulk of the solution.

For the $1.4 \mathrm{~mm}$ thick cells used in the present study, electrode polarization is significant for frequencies below about $100 \mathrm{~Hz}$. The corrected $N$-to- $N^{*}$ - and $N_{D}{ }^{*}$-to- $D_{s}$-transition lines, up to $300 \mathrm{~Hz}$, are given in Fig. 17, where the above given value for $D$ is used. As can be seen, the field amplitudes for both transitions

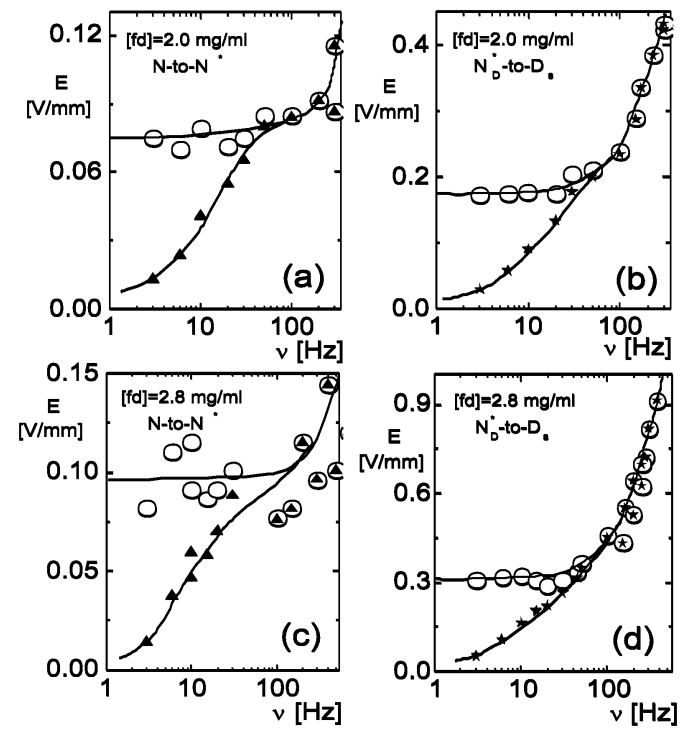

Fig. 17 The corrected $N$-to- $N^{*}$-transition lines (left two figures) and the $N_{D}{ }^{*}$-to- $D_{S}$-transition line (right two figures) for the lower (upper two figures) and higher (lower two figures) fd concentrations. The open circles are the transition lines in Fig. 2 corrected for dielectric polarization only. The filled symbols are data points that are also corrected for electrode polarization. The solid lines are guides to the eye. For frequencies higher than about $100 \mathrm{~Hz}$, electrode polarization for the $1.4 \mathrm{~mm}$ thick cells becomes irrelevant. 
become quite small at very low frequencies, much smaller than the measured values. The corrected phase diagrams in Fig. 17 should be compared to the measured phase diagram in Fig. 2. In any theory that aims at the calculation of the $N$-to- $N^{*}$ - and $N_{D}{ }^{*}$ to- $D_{s}$-transition lines, the corrected phase diagrams in Fig. 17 are the relevant diagrams.

\section{Summary and conclusions}

A number of phases and states can be induced by an external electric field, depending on the field amplitude and frequency. The phase/state diagrams have been constructed by means of polarization microscopy. A more quantitative characterization has been performed for a number of the phases, states and transitions. It is shown that the $N$-phase is a coexistence between isotropic and nematic regions, and it is speculated that the striped texture is a chiral nematic. The pitch of the striped texture is shown to diverge at the $N^{*}$-to- $N$ transition line on lowering the field amplitude. The pitch diminishes at larger amplitudes, and takes the value that has been observed for the chiral texture under high salt conditions in the absence of an electric field. This indicates that the range of the electrostatic interactions decreases with increasing electric field amplitudes. The dynamics of melting and forming of $N$-domains in the dynamical $D_{s^{-}}$and $D_{f}$-states is characterized by means of videocorrelation spectroscopy. The time for melting and reformation of $N$-domains diverges on approach of the $N^{*}{ }_{D}$-phase. Within the $D_{s}$-state this time decreases with increasing field amplitude and remains essentially constant beyond a reasonably sharply defined amplitude, within the $D_{f}$-state. Dynamic light scattering experiments that probe the dynamics on a micrometre length scale reveal a discontinuity at the $N^{*}{ }_{D}$-to- $D_{s}$ transition. This might be due to the abrupt change in microstructural order at this transition due to melting of the chiral texture. Birefringence measurements show that the uniform phase at high frequencies (the $H$-phase) is homeotropically aligned. The orientational order parameter is essentially independent of the field amplitude and frequency, but is a strong function of the fd concentration.

Electrode polarization has to be considered at the low frequencies of interest. A theory is presented for the attenuation of the electric field due to electrode polarization. The theory is tested experimentally and phase diagrams are corrected for electrode polarization. It turns out that electrode polarization, for the typical gap width of our electrical sample cell of $1.4 \mathrm{~mm}$, is important for frequencies below about $100 \mathrm{~Hz}$.

It would also be interesting to investigate the response to electric fields at even lower ionic strengths as well as much higher ionic strengths. Time-resolved confocal microscopy experiments might be helpful to reveal the nature of the dynamic states, and to confirm the 3D chiral structural order of the striped texture. Besides these possible future experiments, theory should be developed. Such a theory should include a description of the deformation of double layers in alternating external fields, the interaction of rods with such deformed double layers (possibly including interactions through electro-osmotic flows), and a prediction of the cooperative behaviour resulting from these interactions.

\section{A. Interpretation of birefringence measurements}

Both for homeotropic and planar alignment of the rods, the refractive index of the suspension is isotropic within the plane parallel to the electrodes. Let $\alpha_{\perp}$ denote the macroscopic polarizability within that plane, the $x y$-plane in Fig. 18a, and $\alpha_{\|}$in the direction perpendicular to that plane, in the $z$-direction.

The incident laser beam makes an angle $\delta$ with the $z$-axis. Within the plane perpendicular to the laser beam, which is the $x y$-plane rotated over an angle $\delta$ along the $y$-axis, the optical axes are along the $y$-direction and along the unit vector $\hat{\mathbf{e}}$, as depicted in Fig. 18b. The polarizability along the $y$-axis is equal to $\alpha_{\perp}$, as before, while the polarizability $\alpha$ along $\hat{\mathbf{e}}$ depends on $\alpha_{\perp}, \alpha_{\|}$and $\delta$. In order to express $\alpha$ in terms of these quantities, consider an electric field $\mathbf{E}$ within the $x z$-plane, as shown in Fig. 18a,b. The electric field has a component $E_{\perp}=E \cos (\delta)$ along the $x$-direction and $E_{\|}=E \sin (\delta)$ along the $z$-direction, where $E$ is the magnitude of $\mathbf{E}$. The corresponding induced dipole moments are,

$$
\begin{aligned}
& \mathbf{P}_{\perp}=\alpha_{\perp} E \cos (\delta) \hat{\mathbf{e}}_{x} \\
& \mathbf{P}_{\|}=\alpha_{\|} E \sin (\delta) \hat{\mathbf{e}}_{z}
\end{aligned}
$$

where $\hat{\mathbf{e}}_{x}$ and $\hat{\mathbf{e}}_{z}$ are the unit vectors along the $x$ - and $z$-direction, respectively. The total induced dipole moment $P$ along the $\hat{\mathbf{e}}$-direction is thus equal to,

$$
P=\hat{\mathbf{e}} \cdot \mathbf{P}=\hat{\mathbf{e}} \cdot\left[\alpha_{\perp} \cos (\delta) \hat{\mathbf{e}}_{x}+\alpha_{\|} \sin (\delta) \hat{\mathbf{e}}_{z}\right] E
$$

Using $\hat{\mathbf{e}}=\cos (\delta) \hat{\mathbf{e}}_{x}+\sin (\delta) \hat{\mathbf{e}}_{z}$, the macroscopic polarizability along the $\hat{\mathbf{e}}$-axis is found to be given by,

$$
\alpha=\alpha_{\perp} \cos ^{2}(\delta)+\alpha_{\|} \sin ^{2}(\delta)
$$

The refractive index $n$ along the $\hat{\mathbf{e}}$-direction is related to the polarizability as $n=\sqrt{\varepsilon_{0}+\alpha}$, where $\varepsilon_{0}$ is the dielectric constant of a vacuum (for convenience we omit the magnetic susceptibility, which is assumed to be that of a vacuum). Let $\bar{n}=\left(n+n_{\perp}\right) /$ 2 denote the average refractive index, where $n$ is the component of the refractive index along the unit vector $\hat{\mathbf{e}}$. We define the average polarizability $\bar{\alpha}$ as $\bar{n}=\sqrt{\varepsilon_{0}+\bar{\alpha}}$. When the difference in

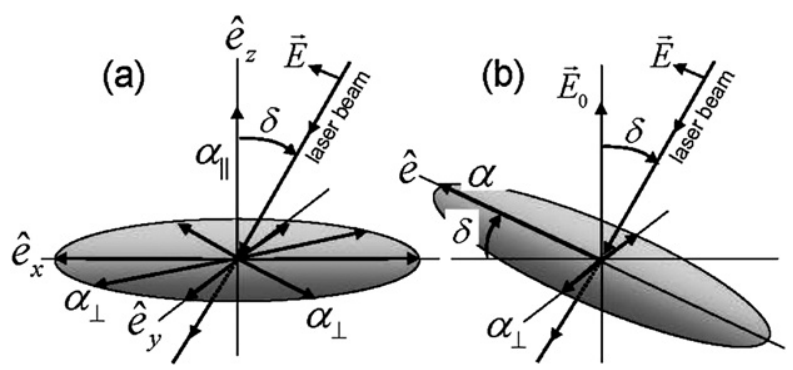

Fig. 18 (a) The polarizability in the $x y$-plane is isotropic and equal to $\alpha_{\perp}$, while in the direction of the external electric field, the $z$-direction, it is equal to $\alpha_{\| \cdot}$. (b) The polarizability that is probed by a laser beam that is incident under an angle $\delta$ is anisotropic in the plane perpendicular to the beam. The polarizability along the $x$-direction is $\alpha_{\perp}$ while it is equal to $\alpha$ along the direction $\hat{\mathbf{e}}$ of the plane spanned by the $z$-axis and the beam direction. 
refractive indices in the two directions is small compared to the average refractive index, we can write,

$$
n=\bar{n} \sqrt{1+\frac{\alpha-\bar{\alpha}}{\bar{n}^{2}}} \approx \frac{\alpha-\bar{\alpha}}{2 \bar{n}}
$$

A similar relation holds between $n_{\perp}$ and $\alpha_{\perp}$. Subtracting these two expressions gives, again up to leading order in differences in polarizability,

$$
n-n_{\perp}=\frac{\alpha-\alpha_{\perp}}{2 \bar{n}}
$$

Substitution of eqn (27) into eqn (29) immediately gives,

$$
n-n_{\perp}=\frac{\alpha_{\|}-\alpha_{\perp}}{2 \bar{n}} \sin ^{2}(\delta)
$$

Similarly we have, $n_{\|}-n_{\perp}=\left[\alpha_{\|}-\alpha_{\perp}\right] / 2 \bar{n}$ where $n_{\perp}$ is the refractive index within the $x y$-plane and, as before, $n_{\|}$in the $z$ direction (see Fig. 18). Hence,

$$
n-n_{\perp}=\left[n_{\|}-n_{\perp}\right] \sin ^{2}(\delta)
$$

This relates the birefringence as measured under an angle of incidence $\delta$ to the true birefringence.

As a second step, the relation between the refractive index difference $n_{\|}-n_{\perp}$ of the suspension must be related to the refractive index difference $n^{\text {rod }} \|-n^{\text {rod }}{ }_{\perp}$ of single colloidal rods along and perpendicular to their long axis. That relation depends on whether the rods have a homeotropic or planar alignment. Here we will only be interested in the sign of the proportionality constant between $n_{\|}-n_{\perp}$ and $n^{\text {rod }}{ }_{\|}-n^{\text {rod }}{ }_{\perp}$. For the determination of the sign of the proportionality constant it suffices to consider perfect alignment.

For the homeotropic case, the rods are aligned along the applied electric field, that is, along the $z$-direction in Fig. 18. In that case we trivially have, $n_{\|}=n_{\|}{ }^{\text {rod }}$ and $n_{\perp}=n_{\perp}{ }^{\text {rod }}$, except for a prefactor that is related to the colloid concentration. For planar alignment, where the rods align with their long axis within the $x y$-plane, with an isotropic distribution within that plane, $n_{\|}$ $=n_{\perp}{ }^{r o d}$, and $n_{\perp}=\left(n_{\perp}{ }^{r o d}+n_{\|}{ }^{r o d}\right) / 2$. Hence, apart from a positive prefactor,

$$
\begin{array}{ll}
n_{\|}-n_{\perp}=n_{\|}^{\text {rod }}-n_{\perp}^{\text {rod }}, & \text { homeotropic } \\
n_{\|}-n_{\perp}=\frac{1}{2}\left[n_{\perp}^{\text {rod }}-n_{\|}^{\text {rod }}\right], & \text { planar }
\end{array}
$$

The homeotropic and planar alignments thus lead to birefringence of opposite sign. The sign of the measured phase shift also depends on the relative orientation of the fast axis of the $\lambda / 4$ wavelength platelet.

\section{B. The analytical form of the free charge density and potential}

The solution of eqn $(13,15,16,17)$ for the free charge density can be written as,

$$
\rho(z, t)=E_{0}\left[R^{\prime}(z) \cos (\omega t)+R^{\prime \prime}(z) \sin (\omega t)\right]
$$

where $R^{\prime}$ and $R^{\prime \prime}$ are the in-phase and out-of-phase response functions for the free charge density, which are equal to,

$$
\begin{array}{r}
\mathrm{R}^{\prime}(z)=A \sin \left(z / l_{0}\right) \cosh \left(z / l_{\mathrm{e}}\right)+\mathrm{B} \cos \left(z / l_{0}\right) \sinh \left(z / l_{\mathrm{e}}\right) \\
R^{\prime \prime}(z)=-B \sin \left(z / l_{o}\right) \cosh \left(z / l_{e}\right)+A \cos \left(z / l_{o}\right) \sinh \left(z / l_{e}\right)
\end{array}
$$

The two length scales $l_{o}>0$ and $l_{e}>0$ are connected to spatial oscillatory behaviour (hence the index " $O$ ") and to exponential decay (hence the index " $e$ ") away from the electrodes, respectively. The oscillatory behaviour of the free charge density is due to the finite propagation time of the charge buildup in the double layer as the applied field changes. These length scales depend on the Debye screening length, the ion-diffusion coefficient and the frequency as,

$$
\begin{aligned}
& l_{e}^{2}=2 \kappa^{-2} \Lambda^{-2}\left[-1+\sqrt{1+\Lambda^{2}}\right], \\
& l_{o}^{2}=1 /\left(l_{e}^{-2}-\kappa^{2}\right)=\left(\frac{D}{\omega}\right)^{2} \frac{4}{l_{e}^{2}}
\end{aligned}
$$

where $\Lambda$ is the dimensionless frequency,

$$
\Lambda=\omega / D \kappa^{2}
$$

Note that $0 \leq l_{e} \leq \kappa^{-1}$ while $l_{o}$ can take any value from 0 for very high frequencies to $\infty$ for zero frequency. Furthermore, the constants $A$ and $B$ are equal to,

$$
\begin{aligned}
& A=\frac{\alpha}{\alpha^{2}+\beta^{2}} \varepsilon \kappa^{2}, \\
& B=\frac{\beta}{\alpha^{2}+\beta^{2}} \varepsilon \kappa^{2}
\end{aligned}
$$

where $\alpha$ and $\beta$ are defined as,

$$
\begin{aligned}
\alpha & =\frac{\Lambda}{1+\Lambda^{2}}\left[\Lambda\left(\frac{p}{l_{o}}+\frac{q}{l_{e}}\right)+\left(\frac{p}{l_{e}}-\frac{q}{l_{o}}\right)+\frac{2}{\Lambda L}(r-\Lambda s)\right] \\
\beta & =\frac{\Lambda}{1+\Lambda^{2}}\left[\Lambda\left(\frac{p}{l_{e}}-\frac{q}{l_{o}}\right)-\left(\frac{p}{l_{o}}+\frac{q}{l_{e}}\right)+\frac{2}{\Lambda L}(s+\Lambda r)\right]
\end{aligned}
$$

and we abbreviated,

$$
\begin{aligned}
p & =\cos \left(L / 2 l_{0}\right) \cosh \left(L / 2 l_{e}\right), \\
q & =\sin \left(L / 2 l_{0}\right) \sinh \left(L / 2 l_{e}\right), \\
r & =\sin \left(L / 2 l_{0}\right) \cosh \left(L / 2 l_{e}\right) \\
s & =\cos \left(L / 2 l_{0}\right) \sinh \left(L / 2 l_{e}\right)
\end{aligned}
$$

Note that the free charge density is essentially zero for distances a few times $l_{e} \leq \kappa^{-1}$ away from the electrodes. According to Poisson's equation, the electric field within the bulk of the suspension, a few times $\kappa^{-1}$ away from the electrodes, is therefore constant, independent of position. The amplitude and phase of this electric field is, however, different from the applied field due to the intervening charges within the (partially developed) double layers near the electrodes.

Similarly to the free charge density, the local electric field $E^{\text {local }}$ is written as,

$$
\begin{aligned}
E^{\text {local }}(z, t) & =-\frac{\partial \Phi(z, t)}{\partial z} \\
& =E_{0}\left[P^{\prime}(z) \cos (\omega t)+P^{\prime \prime}(z) \sin (\omega t)\right]
\end{aligned}
$$

The response functions $P^{\prime}$ and $P^{\prime \prime}$ can be decomposed into contributions $P_{d l}^{\prime}$ and $P^{\prime \prime}{ }_{d l}$ that describe the electric field within the double layers near the electrodes, and contributions $P^{\prime}$ bulk 
and $P^{\prime \prime}$ bulk that describe the field within the bulk, away from the electrodes,

$$
\begin{aligned}
& P^{\prime}(z)=P_{d l}^{\prime}(z)+P_{b u l k}^{\prime}(z) \\
& P^{\prime \prime}(z)=P_{d l}^{\prime \prime}(z)+P_{b u l k}^{\prime \prime}(z)
\end{aligned}
$$

where the double-layer contributions are found to be equal to,

$$
\begin{aligned}
P_{d l}^{\prime}(z) & =\frac{d}{d z}\left[\frac{A+\Lambda B}{\varepsilon \kappa^{2}\left(1+\Lambda^{2}\right)} \sin \left(z / l_{0}\right) \cosh \left(z / l_{e}\right)\right. \\
& \left.+\frac{B-\Lambda A}{\varepsilon \kappa^{2}\left(1+\Lambda^{2}\right)} \cos \left(z / l_{0}\right) \sinh \left(z / l_{e}\right)\right] \\
P_{d l}^{\prime \prime}(z) & =\frac{d}{d z}\left[\frac{\Lambda A-B}{\varepsilon \kappa^{2}\left(1+\Lambda^{2}\right)} \sin \left(z / l_{0}\right) \cosh \left(z / l_{e}\right)\right. \\
& \left.+\frac{A+\Lambda B}{\varepsilon \kappa^{2}\left(1+\Lambda^{2}\right)} \cos \left(z / l_{0}\right) \sinh \left(z / l_{e}\right)\right]
\end{aligned}
$$

while the bulk part of the response functions is given in eqn (19). As for the free charge density, the response functions $P^{\prime} d l$ and $P^{\prime \prime} d l$ are essentially zero for distances a few times $l_{e} \leq \kappa^{-1}$ away from the electrodes, while the response functions $P^{\prime}$ bulk and $P^{\prime \prime}$ bulk describe the electric field in the bulk of the salt solution. Only the latter contributions are of interest for the calculation of the attenuation factor $\gamma$ and the phase shift $\varphi$ of the field that is experienced by colloidal particles in the bulk of the salt solution.

For zero frequency, the above expressions for the free charge density and electric field reduce to,

$$
\begin{aligned}
\rho(z) & =\frac{1}{2} \varepsilon \kappa^{2} E_{0} L \sinh (\kappa z) / \sinh (\kappa L / 2), \\
E^{\text {local }}(z) & =\frac{1}{2} E_{0} L \frac{d}{d z}[\sinh (\kappa z)] / \sinh (\kappa L / 2) .
\end{aligned}
$$

This shows that the electric field is zero at distances a few times $\kappa^{-1}$ away from the electrodes, that is, the double layers act as "perfect insulators".

For finite frequencies, terms like $\sim \sinh \left(z / l_{e}\right), \cosh \left(z / l_{e}\right)$ in the expression (42) for the response functions $P_{d l}^{\prime}$ and $P_{d l}^{\prime \prime}$ for the electric field decay exponentially fast over a distance $l_{e} \leq \kappa^{-1}$ away from the electrodes. These terms are essentially zero in the bulk. Therefore, in the bulk of the salt solution, only the response functions in eqn (19) contribute. The asymptotic expansion of $P^{\prime}{ }_{\text {bulk }}$ and $P^{\prime \prime}$ bulk with respect to large values of $\kappa L$ is somewhat subtle. In order to obtain an explicit expression for the bulk response functions, first of all note that if $\kappa L \gg 1$, the quantity $p$ in eqn (39) is equal to $1 / 2 \cos \left(L / 2 l_{0}\right) \exp \left(L / 2 l_{e}\right)$, up to exponentially small contributions. Similar approximations can be used for $q, r$ and $s$. From eqn (34)-(38) the following expression for the response functions in the bulk of the salt solution is found,

$$
\begin{aligned}
P_{\text {bulk }}^{\prime} & =\frac{4 \Omega^{2}-\Lambda \Omega W^{3}(4-\Lambda \Omega W)}{\Omega^{2}\left(4+\Lambda^{2} W^{4}\right)-8 \Omega \Lambda W^{3}+16 W^{2}} \\
P_{\text {bulk }}^{\prime \prime} & =\frac{-8 \Omega W}{\Omega^{2}\left(4+\Lambda^{2} W^{4}\right)-8 \Omega \Lambda W^{3}+16 W^{2}}
\end{aligned}
$$

where the dimensionless frequency $\Omega$ is defined as,

$$
\Omega=\omega L / D \kappa
$$

and where $W$ is an abbreviation for,

$$
W(\Lambda) \equiv \kappa l_{e}=2^{1 / 2} \Lambda^{-1}\left[-1+\sqrt{1+\Lambda^{2}}\right]^{1 / 2}
$$

Since $\Lambda=\Omega / \kappa L$, the dimensionless frequency $\Lambda$ can be considered a small parameter in the frequency range of interest, where $\Omega \sim 1$, provided that $\kappa L \gg 1$, that is, when the distance between the plates is much larger than the Debye screening length. Since $W \approx 1$ for $\Lambda \ll 1$, this leads to the expressions (20) for the bulk response functions.

\section{Acknowledgements}

We thank Dr. Gögelein for the ALV-DLS test measurements. Part of this research was supported by the Trans-regio SFB 6018 "Physics of Colloidal Dispersions in External Fields" and by the EUFP7 Network "NanoDirect" (contract number CP-FP-213948-2).

\section{References}

1 U. Dassanayake, S. Fraden and A. van Blaaderen, J. Chem. Phys., $2000,112,3851$

2 A. Yethiraj and A. van Blaaderen, Nature, 2003, 421, 513.

3 A.-P. Hynninen and M. Dijkstra, Phys. Rev. Lett., 2005, 94, 138303.

4 F. Mantegazza, T. Bellini, M. Buscaglia, V. Degiorgio and D. A. Saville, J. Chem. Phys., 2000, 113, 6984.

5 H. Kramer, C. Graf, M. Hagenbuchle, C. Johner, C. Martin, P. Schwind and R. Weber, J. Phys. II, 1994, 4, 1061.

6 F. Mantegazza, M. Caggioni, M. L. Jiménez and T. Bellini, Nat. Phys., 2005, 1, 103.

7 X. Schlagberger and R. R. Netz, Europhys. Lett., 2008, 83, 36003.

8 K. Kang and J. K. G. Dhont, Europhys. Lett., 2008, 84, 14005.

9 V. Peikov, S. Stoylov and I. Petkanchin, J. Colloid Interface Sci., 1995, 171, 173.

10 J. Torbet and G. Maret, Biopolymers, 1981, 20, 2657.

11 J. Th. G. Overbeek, Sonderabdruck aus den Kolloid-Beiheften, Band 54, Heft 7-9, 287-364, 1943 (in German).

12 F. Booth, Proc. R. Soc. London, Ser. A, 1950, 203, 533.

13 E. H. B. DeLacey and L. R. White, J. Chem. Soc., Faraday Trans. 2, 1981, 77, 2007.

14 R. W. O'Brien, Adv. Colloid Interface Sci., 1982, 16, 281.

15 M. Fixman and S. Jagannathan, J. Chem. Phys., 1981, 75, 4048.

16 G. S. Manning, J. Chem. Phys., 1989, 90, 5704.

17 C. Chassagne and D. Bedeaux, J. Colloid Interface Sci., 2008, 326, 240.

18 J. Ray and G. S. Manning, Macromolecules, 2000, 33, 2901-2908.

19 G. S. Manning, Europhys. Lett., 2009, 86, 36001.

20 J. Sambrook, E. F. Fritsch and T. Maniatis, Molecular Cloning: A Laboratory Manual, (Cold Spring Harbor Laboratory, New York, 1989).

21 K. Zimmermann, J. Hagedorn, C. C. Heuck, M. Hinrichsen and J. Ludwig, J. Biol. Chem., 1986, 261, 1653.

22 K. Kang, A. Wilk, A. Patkowski and J. K. G. Dhont, J. Chem. Phys, 2007, 126, 214501.

23 K. Kang and J. K. G. Dhont, Eur. Phys. J. E, 2009, DOI: 10.1140/ epje/i2009-10525-4.

24 S. Fraden, Phase transitions in Colloidal Suspensions of Virus Particles, in Observation, Prediction and Simulation of Phase Transitions in Complex Fluids, Ed. M. Baus, L. F. Rull, and J. P. Ryckaert, Kluwer Academic Publishers, Dordrecht, NATO-ASISeries C, vol. 460, 113, 1995.

25 Z. Dogic and S. Fraden, Curr. Opin. Colloid Interface Sci., 2006, 11, 47.

26 Z. Dogic and S. Fraden, Langmuir, 2000, 16, 7820.

27 F. Tombolato, A. Ferrarini and E. Grelet, Phys. Rev. Lett., 2006, 96, 258302.

28 K. R. Purdy, Z. Dogic, S. Fraden, A. Rühm, L. Lurio and S. G. J. Mochrie, Phys. Rev. E: Stat., Nonlinear, Soft Matter Phys, 2003, 67, 031708.

29 M. Scott, R. Paul and K. V. I. S. Kaler, J. Colloid Interface Sci., 2000, 230, 377-387.

30 M. Scott, R. Paul and K. V. I. S. Kaler, J. Colloid Interface Sci., 2000, 230, 388-395.

31 J. D. Ferry, J. Chem. Phys., 1948, 16, 737-738. 